The University of Southern Mississippi

The Aquila Digital Community

Dissertations

Summer 8-2009

\title{
Posttraumatic Spiritual Growth: A Phenomenological Study of Cancer Survivors
}

Ryan Myles Denney

University of Southern Mississippi

Follow this and additional works at: https://aquila.usm.edu/dissertations

Part of the Health Psychology Commons, Oncology Commons, and the Religion Commons

\section{Recommended Citation}

Denney, Ryan Myles, "Posttraumatic Spiritual Growth: A Phenomenological Study of Cancer Survivors" (2009). Dissertations. 1054.

https://aquila.usm.edu/dissertations/1054

This Dissertation is brought to you for free and open access by The Aquila Digital Community. It has been accepted for inclusion in Dissertations by an authorized administrator of The Aquila Digital Community. For more information, please contact Joshua.Cromwell@usm.edu. 
The University of Southern Mississippi

POSTTRAUMATIC SPIRITUAL GROWTH: A PHENOMENOLOGICAL STUDY OF CANCER SURVIVORS

by

Ryan Myles Denney

\begin{abstract}
A Dissertation
Submitted to the Graduate School

of The University of Southern Mississippi in Partial Fulfillment of the Requirements for the Degree of Doctor of Philosophy
\end{abstract}


COPYRIGHT BY

RYAN MYLES DENNEY

2009 
The University of Southern Mississippi

POSTTRAUMATIC SPIRITUAL GROW'TH: A PHENOMENOLOGICAL STUDY OF CANCER SURVIVORS

\author{
by \\ Ryan Myles Denney \\ Abstract of a Dissertation \\ Submitted to the Graduate School \\ of The University of Southern Mississippi \\ in Partial Fulfillment of the Requirements \\ for the Degree of Doctor of Philosophy
}

August 2009 


\begin{abstract}
POSTTRAUMATIC SPIRITUAL GROWTH: A PHENOMENOLOGICAL INVESTIGAT JON OF CANCER SURVIVORS

by

Ryan Myles Denney
\end{abstract}

August 2009

A small but growing body of research has sought to investigate the specific role of religion and spirituality in posttraumatic growth. Recently investigations have begun to focus on spiritual growth following trauma, specifically that of cancer patients and survivors. The purpose of this qualitative study was to investigate how having cancer effects the spiritual growth of cancer survivors across a multidimensional conceptualization of spirituality (Hill 2005; Tsang \& McCullough, 2003). The researcher investigated the lived experience of thirteen cancer survivors with posttraumatic spiritual growth using a phenomenological method of data analysis. Participants reported experiencing spiritual growth across the following domains of spirituality: general religiousness or spirituality, religious or spiritual development, religious or spiritual social participation, religious or spiritual private practices, religious or spiritual support, religious or spiritual coping, religion or spirituality as motivating forces, religious or spiritual experiences, and religious or spiritual commitment. Two novel domains of growth emerged from participants' narratives: evangelism and enhanced spirituality of family/friends. Growth was not endorsed by participants in the following domains of spirituality: religious or spiritual history, religious or spiritual beliefs and values, and religious or spiritual techniques for regulating and reconciling relationships. 


\section{TABI.E OF CONTENTS}

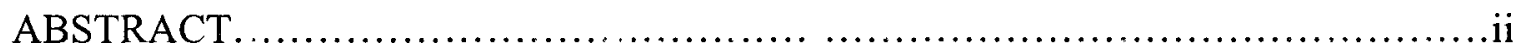

\section{CHAPTER}

I. . INTRODUCTION ................................................

Religion and Coping

Trauma Defined

Posttraumatic Growth

Criticisms and Strengths of Posttraumatic Growth Research

Conceptualizations of Posttraumatic Growth

Posttraumatic Growth Following Cancer

The Role of Spirituality in Posttraumatic Growth

Spiritual Growth Following Cancer

Purpose Statement

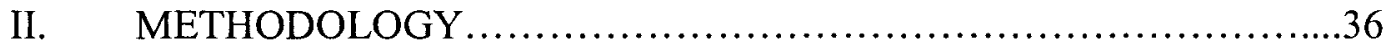

\section{Participants \\ Procedure \\ Instruments \\ Analysis}

III. $\quad$ RESULTS ....................................................49

IV. DISCUSSION ............................................... 68

Implications and Suggestions for Further Research

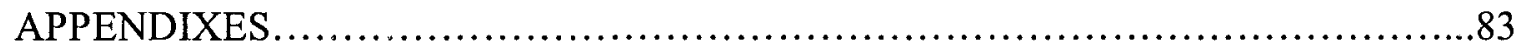

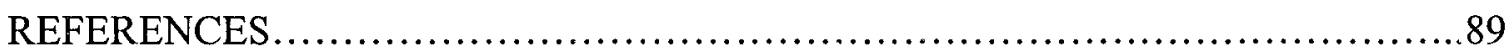




\section{CHAPTER I}

\section{INTRODUCTION}

A growing body of research has sought to investigate the role of spirituality in coping with trauma. Recently, investigations have begun to focus on spiritual growth following trauma, specifically that of cancer patients and survivors. The purpose of this qualitative study was to investigate how having cancer effects the spiritual growth of cancer survivors across a multidimensional conceptualization of spirituality (Hili 2005; Tsang \& McCullough, 2003). First, an overview of conceptualizations of spirituality will be offered, followed by a review of religion and coping and posttraumatic growth research.

Miller and Thoresen (2003) reported that approximately $95 \%$ of Americans profess a belief in God or a higher power, and nine out of ten reported they pray on a daily basis. Sixty-nine percent reported being a member of a local church or synagogue with $40 \%$ reporting that they regularly attend services (Miller \& Thoresen, 2003). In addition, a 2003 Gallup poll reported that $60 \%$ of Americans believe religion is very important in their lives and $62 \%$ believe that religion can answer most or all problems people currently face (Gallup, 2003; see also Gallup \& Linđsay, 1999).

The burgeoning body of research investigating the convergence of religion and spirituality and health seeks to aid psychology in its attempt to ameliorate the pain of traumatic physical and emotional experiences and foster psychological health. Religious and spiritual experiences are often used by people to cope with distressing events. Before describing the religion and health research, it is relevant to first define and distinguish the 
terms "religion" and "spirituality," as well as three main orientations to religious experience, and the potential positive and negative aspects of religion and spirituality.

\section{Definitions of Religion and Spirituality}

The definition of spirituality has been elusive and a source of much debate among researchers (Paloutzian \& Park, 2005; Pargament, 2002). Historically, religion has been defined in terms of symbclic, codified practices that aim to interface with the sacred, while spirituality has been thought of as an individualized, personal search for meaning and the transcendent. Stated differently, spirituality has been defined as the personally experienced, subjective facet of religious experience, whereas religion is often defined as a formal set of rules and practices pertaining to and often regulating spirituality (Hill \& Pargament, 2003). Religion is viewed as systematic, doctrinal, and authoritative, while spirituality is seen as more inward, personal, spontaneous, private, universal, and emotional (Hill \& Pargament, 2003; Richards \& Bergin, 2005). Religion is also characterized by a focus on community, orthodoxy, institutional practices, an authoritarian hierarchy, and specific doctrine regarding good and evil, whereas spirituality is characterized by a focus on the individual, informality, emotionally oriented practices, a lack of formal accountability, and an emphasis on universalism over doctrine (Koenig, McCullough, \& Larson, 2001).

More recently, Zinnbauer and Pargament (2005) further described spirituality as "a personal or group search for the sacred," and religiousness as "a personal or group search for the sacred that unfolds within a traditionally sacred context" (p. 35). Religion can be thought of as a context in which spirituality is often expressed, but the two concepts may be intertwined or experienced as distinctly separate. Further, Richards and 
Bergin (2.005) point unt tiut it is possible to be religious without necessarily being spiritual and sprritual withoul rigid adherence to a specific religion. Neither should be considered more important, but rather they should be conceptualized as two equally valid, yet.distinct modes of experience. Lespite the distincrion between religion and spirituality described in the literature, the terms are still sometimes used concurrently as an acknowledgement of their interconnectedness.

Domains of Religion and Spirituality

A number of instruments have been developed to measure religious and spiritual experience, but due to the multifaceted nature of religion and spirituality, there is not currently a "best measure" that adequately captures their entire scope (Tsang \& McCullough, 2003). Overall, the majority of instruments only measure one facet of religiosity or spirituality. For example, religious belief, commitment, affiliation, development, maturity, and well-being can all be measured with relative reliably (Hill, 2005). But a single, comnibus measure of religiousness has proven extraordinarily difficult to construct. As a resuit, Tsang and McCullough (2003) proposed a Hierarchical Model of spirituality consisting of two levels or domains. The model has been offered in an attempt to facilitate the measurement of spirituality, and to address the complex nature of this construct.

Specifically, the Hierarchical Model of spirituality can be divided into a dispositional level (Level I) and operational level (Level II). The dispositional level seeks to describe religious traits or the characteristics of persons more likely to be religious. Furthermore, this level is tied to the idea that a personality trait predisposing people to an interest in religious pursuits exists, and that this trait is independent from the Big Five 
personality dimensions (Tsang \& McCullough, 2003). The operational level describes the diversity with which spirituality is expressed and experienced, as well as how one's spirituality manifests in thè real-world of daily living (Tsang \& McCullough, 2003). For example, two persons who are equaily disposed toward spirituality at the dispositional . level may have very different ways of expressing and experiencing their spirituality, including differences in spiritual orientation (intrinsic, extrinsic, quest), types of spiritual coping, and the various uses and experiences of prayer. Building on the work of Tsang and McCullough (2003), Hill (2005) further delineated twelve domains of religiousness and spirituality under these two levels. Three of the domains outlined by Hill were researcher generated, while the remaining nine were derived from The Fetzer Institute/National Institute of Aging Working Group (1999). Domains conceptualized at the dispositional level include: (a) general religiousness or spirituality (domain one): seeks to describe a person's overall religiousness and the personality traits that may predispose a person to increased spirituality; (b) religious or spiritual commitment (domain two): describes the degree to which a person is committed to his or her religious beliefs or tradition; (c) religious or spiritual development (domain three): refers to the level of maturity a person has reached in relation to his or her religious or spiritual belief system; (d) religious or spiritual history (domain four): has been measured by scales seeking to quantify the degree to which persons have a family heritage of religious faith. Domains conceptualized at the operational level include: (a) religious or spiritual social participation (domain five): refers to the amount of explicit religious social support, religious involvement, and attitude toward a faith community; (b) religious or spiritual. private practices (domain six): describes the degree to which people participate in their 
religion or practice rituals in their personal lives, such as prayer, meditation, and reading sacred documents; (c) religious sr spiritual support (domain seven): refers to the degree to which people experience emotional and spiritual support from others of their faith; (d). religious or spiritual coping (domain eight): is how often and in what way religious coping strategies are used to deal with stressful life events; (e) religious or spiritual beliefs and.values (domain nine): attempts to tap the extent of a person's fundamentalistic or orthodox religious or spiritual beliefs. That is, this domain seeks to gauge how strict a person's faith system is and how rigidly adherent they are to this faith system; (f) religion or spirituality as motivating forces (domain 10) focuses on how much peoples' spiritual beliefs motivate their behavior, cognitions, and emotions; (g) religious or spiritual techniques for regulating and reconciling relationships (domain 11) refers to how one's spirituality affects how relationships are managed, especially negative relational aspects; (h) religious or spiritual experiences (domain 12) refers to positive and negative types of religious experiences. Such positive experiences may include a sense of involvement with the transcendent or finding meaning in life, while examples of negative experiences may include religious strain (Exline \& Rose, 2005), struggles with God and others, or intrapersonal anxiety (Hill, 2005). While there are quantitative measures that seek to tap each of these domains individually (Hill, 2005), all of the domains have not to date been used together in a single investigation. This 12 domain model of spirituality will be used as the theoretical underpinning of the present study as it offers the most comprehensive, detailed conceptualization of spirituality described in the literature to date, and the spiritual experience participants will be investigated across all 12 domains. Intrinsic, Extrinsic, and Quest Orientations 
In addition to specific domains associated with religion and spirituality, three primary religious and spiritual orientations that describe how people approach the sacred have been identified. Allport and Ross (1967) identified and distinguished between intrinsic and extrinsic orientations to reilgiousness, which imply that people have varied motives for seeking religious experiences and contexts. Intrinsic religiousness is characterized by a personal search for spirituality. Intrinsically religious persons make every effort to internalize their beliefs and to bring their personal lives into congruence with those beliefs. Intrinsically oriented persons also tend to primarily focus on God with a secondary, even negative view of the self. Conversely, extrinsically oriented persons tend to focus primarily on the self, with less internal emphasis on the sacred. Individuals with this orientation tend to use religion to meet personal needs, such as using religion to find security, interact socially, attain status, distract from life difficulties, or buffer anxiety. Extrinsically oriented persons tend to focus on religious practices and customs with a secondary focus on a personal search for the divine.

As an alternative to Allport's intrinsic and extrinsic orientations, Batson; Schoenrade, and Ventis (1993) proposed the quest orientation. This orientation consists of "an approach that involves honestly facing existential questions in all their complexity, while at the same time resisting clear-cut, pat answers" (Batson, Schoenrade, \& Ventis, 1993, p. 166): Persons with a quest orientation to religiousness believe that it may be impossible to know the ultimate truth about existential matters, but that such questions are important to the human condition. Though current religious questions themselves are viewed as tentative and subject to alteration, answers are continually sought. (Batson, 
Suhoenrade, \& Ventis, 1993). Researchers have found that the quest approach is a unique orientation, distinctly separate from the intrinsic and extrinsic orientations.

Negative Effects of Religion/spirituality

Historicaily, mentai health proressionais have heid a negative view of religion and spirituality (Richards \& Bergin, 2005), sometimes believing religion and spirituality to be a source of more harm than good, and that rigid religious beliefs stifle cognitive flexibility and a healthy view of seif. Several pioneers of psychological thought have viewed religion as having negative effects on mental and physical health (Richards \& Bergin, 2005). For example, Sigmund Freud, the father of modern psychotherapy, called religion "the universal neurosis of humanity," while emphasizing that "in the long run nothing can withstand reason and experience, and the contradiction which religion offers to both is all too palpable" (Freud, 1927, p. 43). Albert E1lis (1980), who developed Rational Emotive Therapy, proposed that religion blocks mental health via the discouragement of self-acceptance and self-directedness. While it is worth noting that Ellis has since'modified his view, he asserted that religion tosters intolerance of others, difficulty with the acceptance of ambiguity, and irrational (versus scientific) thinking (Koenig, McCullough, \& Larson, 2001).

More modern researchers have pointed to the concern that some religious beliefs may disccurage or keep people from seeking medical or mental health services in a timely fashion. Additionally, a concern has been voiced that religious beliefs may prevent persons from complying with treatment recommendations, relying on research data, or seeking life-saving medical interventions (Koenig, McCullough, \& Larson, 2001). Fxline, Yali, and Sanderson (2000) found religious strain (e.g., viewing God as distant or 
punishing) to be a major predictor of psychological distress, specifically depression and suicide. Feelings of alienation were further strongly associated with depression, especially in the clinical sample. Moreover, interpersonal conflict with religious figures . and internal dissonance surrounding church teachings has been linked with experiencing an increase of negative emotions (Pargament et al., 1998). Additionally, Hathaway. (1999) has proposed that psychopathology can have a detrimental impact on religious and spiritual functioning and well-being. Some have called for this religious impairment to'be included in future revisions of the Diagnostic and Statistical Manual of Mental . Disorders, as empirical support exists showing a positive correlation between mental health and both religious and spiritual orientations and behaviors (Loewenthal, 1995; Yarhouse, 2003).

Psychological distress has also been connected to religion that is rigid, fundamentalistic, and prejudice (Pargament, 2002). For example, religious fundamentalism has received a great deal of attention in the literature and has been . strongly linked to negative mental health outcomes. The term "fundamentalism" refers to . ' religious practices and beliefs that (Koenig, McCullough, \& Larson, 2001, p. 55) relate to traditional, immutable beliefs of a religious system. Hood, Hill, and Williamson (2005) have cautioned against adopting too broad a definition of fundamentalism, as the term has come to be misused, sometimes being used as a synonym for bigetry, fanaticism, or antiintellectualism. Indeed, these authors assert that fundamentalism is more than merely a. label for certain religious beliefs, it is "an approach to life with cognitive and affective components through which people give religious meaning to their experiences and rigidly adhere to the teachings of their faith" (Hood, Hill, \& Williamson, 2005, p. 5). Rigid 
religious fundamentalism has been strongly linked to prejudice, narrow mindedness, intolerance for differences, discrimination, and an increased willingness to endorse the use of force to perpetuate one's ideas (Pargament, 2002). For example, Altemeyer and Hunsberger (1992) reported a significant relationship between fundamentalism and participants' willingness to support the arrest, torture, and execution of political radicals. Additionally, religious fundamentalism has been linked to prejudiced attitudes toward persons of another religious faith, women, and homosexual persons (Hunsberger, Owusu, and Duck 1999; Rowatt, Franklin, \& Cotton, 2005). In sum, religious fundamentalism has been closely associated with negative psychological health and social justice outcomes.

Positive Effects of Religion/Spirituality

Psychological well-being has been associated with religion that is internalized, intrinsically motivated, and based on a secure relationship with God (Pargament, 2002). More highly religious persons are more likely to stay married (Cohen et al., 1997), are less likely to abuse alcohol and drugs (Gorsuch \& Butler, 1976; Stark, 1996), often have increased levels social support (Bradley, 1995; Ellison \& George, 1994), tend to be more optimistic (Sethi \& Seligman, 1994) and hopeful (Plante \& Boccaccini, 1997; Ringdal, 1996; Sethi \& Seligman, 1994), and are more inclined to have a fulfilling sense of purpose or meaning in life (Bolt, 1975, Carroll, 1993). Research has found high levels of religiosity/spirituality to be associated with well-being (Koenig, 1997; Larson et al., 1997), as well as potentially buffering against the negative effects of divorce, drug abuse, physical illness, some forms of stress, depression, and suicide (Exline, Yạli, \& Sanderson, 2000). 
. Religion has also proven especially helpful for groups that are socially

marginalized, often providing a cohesive belief system and a platform from which to . speak (Pargament, 2002). Increased religious support has been linked to less depression in bereaved parents (Maton, 1989). A1so, in a national sample of older persons, Krause (1998) found religious coping was protective against the negative psychological effects of living in a deteriorated neighborhoed. Evidence also suggests that the more integrated ... a person's religious beliefs, practices, relationships, and motivations the more likely he or she will be to experience the positive effects of religion. If religious beliefs and practices become fragmented, contradictory, or nonintegrated, psychological dissonance and uncomfortable levels anxiety are likely to result (Pargament, 2002).

\section{Religion and Coping}

“Religion might be particularly valuable to people when they are facing problems that push them to the limits of their own personal and social resources" (Pargament, 2002, p. 176). When faced with stressful life events, many people turn to their faith for comfort, support, and a sense of meaning and control (Pargament \& Ano, 2006). Most commonly, researchers have found that people use prayer, worship, and social support from a faith community to cope with suffering (Pargament, 2005; Tatsumura, Maskarinec, Shumay, \& Kakai, 2003). Pargament (1997), for example, has compiled a lengthy, rather diverse list of studies that seek to indicate the degree to which several different groups use religion to cope. A study of 586 members of mainline Christian churches in America revealed that $78 \%$ of church members utilized religion in their coping with difficult life circumstances (Pargament et al., 1990). A study of 1,000 battered wives revealed that one-third sought help from clergy (Bowker, 1988). Of 1,299 
Afficar Americans, $80 \%$ reported using prayer to cope (Ellison \& Taylor, 1996).

Similarly, Kesselring, Dodd, liatsey, and Strauss (i986) reported that $37 \%$ of Swiss respondents and $92 \%$ of Egyptian respondents believed that God would help them through their cancer-reiated iilness. Koenig, George, and Siegler (1988) also reported that $45 \%$ of older, Protestant adults believed religion to be an important part of coping. In short many pecple turn to religious leaders and practices to cope with traumatic or painful circumstances.

In addition to group membership, there are other factors, or personal predictors that serve to influence the probability a person will use religion in coping (Pargament 1997). Individual demographic differences have been found to predict utilization of spirituality after trauma. For example, one of the strongest and most common findings of several studies was that women and people who are less educated are most likely to report the use of prayer and religion as a coping mechanisms (Bearon \& Koenig, 1990; Bjorck \& Cohen, 1993; Ellison \& Taylor, 1996; Gurin, Veroff, \& Feld, 1960). Ferraro and Koch (1994) reported that religious coping was also more frequent among older, African American married participants, and they observed no relationship between socioeconomic status and religious coping. Overall, a person's gender, religious affiliation, level of education, and ethnicity tend to be important factors in the use religion to cope with major life stress or trauma (Pargament, 2002).

Styles of religious coping. Three primary approaches to religious coping have been identified (Pargament et al., 1988; Pargament, 2002). The self-directed approach refers to people relying on their own internal resources to cope, believing those resources to be God-given. The deferring approach refers to people passively transferring 
responsibility for problem solution to God. In the collaborative approach, people view . themselves as partnering with God to solve problems. Pargament $(1988,2002)$ also : describes several correlates of these approaches. The self-directed approach to coping.has. been linked to higher seif-esteem and an increased sense of control, while the deferring ", : approach has been connected to lower self-esteem, external locus of control, poorer problem-solving skills, and increased intolerance for human diversity. Collaborative religious coping, which involves the person and God reciprocating responsibility, has been associated with higher self-esteem, and internal locus of control, and has been described by several researchers as the most psychologically healthy method of religious coping.

Pargament (1997) further described positive religious coping, citing that many people believe that God is guiding and supporting them through their times of trouble. Research has shown that those who experience spiritual support often report more positive outcomes (Pargament, 2005; Pargament, 1997; Tatsumura, Maskarinec, Shumay, \& Kakai, 2003). Churches and synagogues are used more than any other support system as a source of social support in times of distress. People sometimes describe their faith communities as second families as they rely on the financial, emotional, and spiritual support of members and clergy. This is an especially salient point in light of the cogent empirical evidence that social support is positively related to healthy coping. Additionally, positive religious reframing has been linked to better outcomes. Persons who attribute death, illness, or other major losses to the will of God or to a benevolent God are more likely to experience positive coping, as are persons who frame their experience as an opportunity to grow spiritually (Pargament \& Ano, 2006). Likewise, 
cancer patients who attributed more control of their illness to God have reported higher self-esteem and better adjustment (Jenkins \& Pargament, 1988). Tarakeshwar et al. (2006) also found that positive religious coping was related to better overall quality of life in persons with advanced cancer.

Negative religious and spiritual coping mechanisms, which occur when religion and spirituality play a role in coping that is not health-promoting (e.g., feeling abandoned by God) have also been identified. Pargament (2005) reported that:

Those who made more use of negative religious coping strategies were more likely to experience unfavorable physical and psychological outcomes, such as greater physical impairment in daily activities, lower cognitive functioning, more symptoms of depressed mood, and lower quality of life (p. 677).

Major medical illness, for example, may lead to more distress and even physical symptoms because the illness itself represents a threat to one's foundation of faith (Pargament \& Ano, 2006). McConnell, Pargament, Ellison, and Flannelly (2006) found that negative religious coping was significantly linked to various forms of psychopathology, including general anxiety, phobic anxiety, depression, paranoid ideation, obsessive-compulsiveness, and somatization. Also, the relationship between negative religious coping and various forms of anxiety was more pronounced for persons who had recently experienced a serious illness such as cancer (McConnell, Pargament, Ellison \& Flannelly, 2006). Moreover, faith communities while often a source of support, can also be a source of psychosocial anxiety (Pargament, 1997). Sometimes distressed persons report feeling abandoned by church members and leaders, or they feel they have been a disappointment to their faith community. This can have a negative impact on 
coping, which leads to feelings of hopelessness, despair, and resentment. Just as there is positive religious reframing, there can be also he negative reframing. Seeing one's trauma as a deserved punishment from God is the most common negative religious reframe, the effect'of which is often to stifie adaptive coping (Fargament, 1997).

\section{Trauma Defined}

The word trauma comes from the Greek word meaning wound. Researchers have described "trauma" along on a continuum ranging from specific symptoms and events to broader, overarching conceptualizations (Park, 2004). The Diagnostic and Statistical Manual of Mental Disorders (2000) describes Posttraumatic Stress Disorder and Acute Stress Disorder both of which may result from "exposure to an extreme traumatic stressor...that involves actual or threatened death or serious injury, or other threat to one's physical integrity" (p. 463). To meet criteria for one of these disorders, a person's response to the traumatic stressor must be characterized by "intense fear, helplessness, or horror" (p. 467/471). Trauma research often describes trauma in this way (Park, 2004), but some posttraumatic growth researchers have chosen to expand upon this definition. For example, Calhoun and Tedeschi (2006), offer a more broad conceptualization of a traumatic experience, defining it as: (a) a set of circumstances that significantly challenge or invalidate the person's preexisting assumptive world, (b) an event that disrupts a "person's personal narrative or the meaning they give to life events, (c) a "watershed" event that divides a life into before and after the event, and (d) an experience of loss, grief, suffering or other negative event that causes a transformation in the view of self, others and philosophy of life. These researchers further postulate that though people may 
endure'similar experiences, be cutcone can be quite :aried (e.g., one labeling an event iraumatic, the other nen-traurnatic).

- Traumatic events can be further categorized based on several characteristics of the event. The.Sidran institute for Traumatic Stress Education and Advocacy (2006) (an established, non-profit organization whose goal is the advancement of trauma research) categorizes traumatic experiences as either single blow or repeated trauma. Single blow, trauma is characterized by a trauma inducing event that occurs only one time. Examples of single blow trauma include natural disasters (e.g., earthquakes, tornados, hurricanes), technological disasters (e.g., auto/plane crashes, chemical spills), and criminal violence (e.g., robbery, rape, homicide). While single blow events can be extraordinarily distressing, when trauma is experienced repeatedly, the psychological effects can be more harmful and longer lasting since loss of psychological resources has been shown to account for a large portion of the variance in psychological distress (Sattler et al., 2006). Examples of repeated blow trauma may inciude ongoing abuse, chronic disease, and multiple significant losses. Traumatic experiences can be either natural (e.g., natural disasters, disease) or man-made (e.g, ierror attacks, abuse). Overall, the research suggests that man-made trauma can be much more difficult for people to deal with because it causes longer lasting and deeper emotional pain (Sidran Institute, 2006). The mest debilitating negative psychological effects have been found when the injury is induced by a person on whom the victim is dependent (e.g., abuse).

Cancer as trauma. Some researchers have also argued that health-related problems such as being diagnosed with cancer can lead to both physical and psychological trauma (e.g., Erickson \& Steiner, 2001; Stanton, Bower, \& Low, 2006). 
According to Stanton, Bower, and Low (2006), a cancer diagnosis challenges the core assumptions of an individual as well as one's self-esteem and sense of control or efficacy: The National Cancer Institute (2007) defines trauma as "injury to the body, or an event that causes long-lasting mental or emotional damage." Having cancer involves both aspects of this definition - threat to body and threat to psyche and is clearly a stressful experience with the potential for producing negative psychosocial outcomes (Cordova, Cunningham, Carlson, \& Andrykowski, 2001). This definition is also consistent with both the descriptions of trauma elucidated by both the DSM (DSM-IV-TR, 2000) and posttraumatic growth researchers (e.g., Calhoun and Tedeschi, 2006). Many psychological researchers take for granted the fact that having cancer is traumatic, often calling cancer a life-threatening illness (Holland et al., 1999), or referring to their research as part of the ongoing battle with cancer (Kaplar, Wachholtz, \& O'Brian, 2004). Also, when studying the effect of cancer on life variables, researchers have investigated several domains including (a) perceived stressfulness of having cancer, (b) disease severity, (c) degree of psychological distress caused by the cancer experience, and (d) the impact of cancer upon subjective well-being and quality of life (Stanton, Bower, \& Low, 2006). The way in which cancer is viewed and studied by the scientific community is . indicative of the fact that it is often viewed as a traumatic experience (Erickson $\&$ Steiner, 2001). A diagnosis of cancer would also appear to fit the aforementioned characteristics of trauma elucidated by the Sidran Institute, considering that the most. psychologically distressing traumas are repeated blow, unpredictable, and multifaceted (Erickson \& Steiner, 2001; Greenstein \& Breitbart, 2000; Hantman \& Solomon, 2007). Cancer is potentially a repeated blow trauma, in that it is not uncommon for a person to 
experience periods of remission, only to have the cancer reemerge. The disease can be unpredictable, may emerge anywhere in the body, and cannot always be cured. Moreover, several forms of cancer can reemerge with a vengeance or metastasize, making the repeated blow even more difficuit to deal with. It is multifaceted in that in addition to being a medical disease, it can also bring with it social, personal, spiritual, and familial uncertainty (Cordova, Cunningham, Car'son, \& Andrykowski, 2001).

\section{Posttraumatic Growth}

According to Tedeschi and Calhoun, posttraumatic growth can be defined as "positive psychological change experienced as a result of the struggle with highly challenging life circumstances" (Tedeschi \& Calhoun, 2004, p. 1). Human psychological coping processes seem to have the capacity to use traumatic experiences as a means for growth. That is, while it may seem counterintuitive, traumatic experiences can foster positive change. Posttraumatic growth is sometimes referred to in the literature as benefit finding, stress-related growth, perceived benefits, or positive change (Janoff-Bulman, 2004).

The idea that life difficulties can lead to growth in humans is not new. This idea has pervaded literary, philosophical, mythical, and religious thinking for thousands of years. Many major world religions, including Christianity, Hinduism, and Islam, consider suffering crucial to the development of wisdom, as well as to the cultivation of . relationships with others and a higher being (Shaw, Joseph, \& Linley, 2005). Many of these faiths view pain as necessary for purification, holiness, or preparation for life to come (Calhoun \& Tedeschi, 2006). What is relatively recent, however, is the scholarly attention posttraumatic growth has begun to receive from researchers in the fields of 
psychology, counseling, psychiatry, and social work. Within the last two decades, both qualitative and quantitative methodologies have been employed to systematically investigate positive, trauma-stimulated change (e.g., Cadell, Regehr, \& Hemsworth, 2003; Calhoun \& Tedeschi, 2006; Manne et al., 2004; Tomich \& Helgeson, 2004; Weiss, 2004). As noted earlier, traumatic events can cause severe negative psychological consequences for people. Yet, there is also compelling data to suggest that, for many people, an encounter with a negative life event can also lead to a positive psychological outcome (Park, Cohen, \& Murch, 1996; Calhoun \& Tedeschi, 2006).

\section{What Constitutes "Growth?"}

Two important issues associated with posttraumatic growth involve questions about the definition and parameters of growth (Park \& Lechner, 2006), and how we can know that a trauma survivor has indeed experienced "growth." Calhoun and Tedeschi (2006) have been quick to assert that, due to the wide variety of experience and the extreme nature of individual differences, there can be no one, all encompassing definition of posttraumatic growth. 'They warn against assuming positive growth in every person who experiences trauma as well as assuming any change at all. Moreover, when attempts are made to measure posttraumatic growth quantitatively, there is ongoing debate over what cut-off scores indicate true growth. The process of deciding whether change in a participant was sufficiently positive to be labeled as posttraumatic growth can be difficult and potentially arbitrary (Calhoun \& Tedeschi, 2006). Most agree that this question can be truly answered only by the individuals who have experienced the trauma (Park \& Lechner, 2006; Wortman, 2004). 
Despite research supporting postirairatic grow th, the concept has not gone without scrutiny. To approach the ressorch of trauma with the assumption that survivors have experienced growth, an idea that has streng ties to the positive psychology movemeni, has been viewed by some'as overiy optimistic (e.g., Wortman, 2004). Some researchers believe that in seeking to understand posttraumatic growth current research has become biased teward positive cliangss (Furk \& Lechner, 2006; Wortman, 2004). Another criticism is that participants are asked to describe only positive changes they have experienced as a result of a trauma as opposed to being asked whether they have experienced any changes at all. Undoubtedly, this approach biases the nature of the data collected (Park \& Lechner, 2006). Moreover, it has also been found that research participants are less willing to disclose negative changes associated with trauma. McMillen, Zuravin, and Rideout (1995) found that, and when given an open-ended question, approximately half of the participants reported some benefit. This finding could be the result of positive impression management, or participants' desire to downplay the negative in order to cope, or a reflection of the participants' search for a purpose or meaning in their pain (Wortman, 2004).

Despite the aforementioned criticisms, researchers have continued to give attention to the positive psychological aspects of traumatic events. Many researchers have scught to mitigate the effects of positive impression management by inviting participants to describe both the positive and negative impacts of stressful events, but this need not preclude researchers from specifically emphasizing the positive (Park \& Lechner, 2006). Many people report having learned highly valuable lessons from their traumatic experiences, which, they assert, have aliowed them to have made them better able to live 
more fulfilling lives. The variables associated with this growth are of interest to clinicians, who are working to enhance well-being (Calhoun \& Tedeschi, 2006). The proponents of posttraumatic growth assert that this line of inquiry should be pursued . because such growth is reported with high frequency by survivors and has the potential to enhance mental health (Smith \& Cook, 2004), increase a sense of meaning or purpose in life (Janoff-Bulman, 2004; Neimeyer, 2006), and foster human resiliency (Dienstbier, 1989; Lepore \& Revenson, 2006). Most researchers recognize that traumatic experiences have both positive and negative outcomes, but they seek to fill a gap in the literature by studying both the way in which positive growth takes place and its consequences. (Tedeschi \& Calhoun, 2004). Finally, posttraumatic growth research is consistent with the values of the positive psychology movement, which emphasizes optimism, hope and the human capacity for positive growth (Seligman, 2005).

\section{Conceptualizations of Posttraumatic Growth}

While research on stress-related growth is a growing area of inquiry in the social and behavioral sciences, there remains a dearth of literature describing comprehensive models of posttraumatic growth (Park, 1998; Park \& Ai, 2006). Tedeschi and Calhoun (1995) have proposed the most detailed model to date. These current growth conceptualizations are in their infancy and are thus decidedly general and abstract, and empirical evidence for them is almost nonexistent. However, these hypotheses do share threads of commonality. For example, across theories, there are two main conditions that are considered necessary in order for posttraumatic growth to be catalyzed. The first condition is that the perceived impact of the stressor must be great (Stanton, Bower, \& Low, 2006). Yet, there seems to be a critical mass or subjective internal threshold beyond 
which cognitive coping resources are overwhelmed and positive growth becomes less . likely (e.g., when an individual's psychological resources are overwhelmed). A second commonality is intentional engagement with the stressor by the person experiencing the trauma. Deliberate cognitive processing, affect management, self-disclosure, renewed meaning-making, actively seeking social support, and approach-oriented coping are all components of intentional engagement (Stanton, Bower, \& Low; 2006).

Preliminary conceptualizations of posttraumatic growth have involved descriptions of the importance of meaning-making following a traumatic experience. For example, similar to Meichenbaum's (1985) stress inoculation model, Janoff-Bulman's (2004) postulated that survivors rebuild their assumptive worlds in a way that makes them more resilient to future assaults. Having integrated lessons learned from their experience, survivors reconstruct their inner world (e.g., reorder their priorities). The main reason traumatic experiences are often so difficult to endure is that most people do not expect tragedy to hit them personally, and they are thus unprepared to handle this tragedy (Janoff-Bulman, 2004). Survivors who have assimilated life's unpredictable nature and their own fragility into new schemata are thought to have a more realistic, psychologically heaithy cognitive mechanism to face pain (Davis, Wohl, \& Verberg, 2007; Janoff-Bulman, 2004). This is supported by the finding that cognitive adaptation following a traumatic experience and reappraising the stressor as positive has been found to be crucial to healthy psychological coping (Park, 2005). Moreover, Davis and colleagues (2007) found that survivors of loss who confronted and resolved their crisis of meaning were more likely to report positive growth. 
Some theorists have sought to describe more specifically the characteristics of this meaning-making process. Jainff-Bulman (2004) suggests that growth involves constructing new conceptualizations of the self in the aftermath of stressful events as survivors often become aware, for the first time, of strengths and abilities within themselves that they would not have discovered had they not experienced their trauma. Survivors develop and discover new psychological coping skills and resources that can lead to new life possibilities. For example, Janoff-Bulman (2004) interviewed a newly paralyzed athlete who praised a newfound "life of the mind" he discovered through reading This is consistent with idea and observation of several researchers and clinicians that positive meaning-making is a critical component of psychological resilience and growth (Bower et al., 1998; Frankl, 1969; Park, 1998). Indeed, events are often experienced as traumatic because they violate a person's ability to construct positive meaning or they disrupt a person's personal meaning-making (Park \& Ai, 2006). Positive meaning-making has been shown to reduce the negative effects of traumatic experiences. For example, Ai and her colleagues (2005) sampled 457 college students following the September 11 terrorist attacks and found that a greater sense of spiritual meaning was a protective factor against depression and anxiety. Boeschen and colleagues (2001) also found that rape survivors who avoided making cognitive and emotional sense of their traumatic experiences and memories had higher levels of PTSD. Moreover, survivors of extreme trauma often report an increased appreciation for life and a renewed appreciation for one's existence, with significant relationships being of particularly renewed importance (Calhoun \& Tedeschi, 2006). In short, survivors often cherish life with revitalized energy. Since people tend to inherently seek meaning in life and experience, 
approaches to the treatment of iraima survivors that involve new meanirg-making fe.g., narrative therapy, cogntive restru turing) have provel most promising (Park \& Ai, 2006).

\section{Calhoun and Tedeschi's Modei}

In describing the most extensive model of posttraumatic growth offered to date, T'edeschi and Calhoun (1995) identified three overarching domains or categories of such growth. Factor analysis cn subsequent data revealed five orthogonal sub-factors of change. There can be changes within a person that go beyond these common core domains, or that are specific to the type of trauma the person has experienced (e.g., healthier eating following a cancer diagnosis). The core domains along with their accompanying sub domains are described below.

Changed perception of self. The first core domain is a changed perception of the self, accompanied by the sub-domains of strength and new possibilities. Persons who hiave survived a iraumatic experience often begin to see themselves as "vulnerable, yet stronger" (Caihoun \& Tedeschi, 2006, p. 5). The survivor comes to see, often in stark reality, that his or her own mortality and the fragility of life. The world is seen as a dangerous and unpredictable place, yet an inner strength may develop. Persons often begin to look hopefully toward future challenges and traumas, believing that, if they can handle what they have already erdured, they will be able to manage any new trauma that may come. Also, some people experience the emergence of a new focus for their lives. New doors of possibility open to them as they seek new life paths that they never would

$\therefore$ have considered before experiencing their trauma (Calhoun \& Tedeschi, 2006). 
Relating to others. The second dornain of posttraumatic growth involves the way in which the survivor relates to others. Oftentimes a consequence of coping with a personal traumatic experience is a changed view of humanity as a whole. Survivors have reported an increased sense of connection with other people in general, as well as an increased sense of compassion for others who are suffering, and a greater connection to the "living" (Calhoun \& Tedeschi, 2006). Survivors also report a greater sense of . wholeness and comfort within themselves when relating to others. Persons who have struggled with traumatic life events often report increased intimacy with others, greater freedom to be oneself, and increased willingness to self-disclose even socially undesirable elements of one's self or experience (Calhoun \& Tedeschi, 2006).

Changed philosophy of life. A third domain of posttraumatic growth involves a reevaluation of one's values and priorities. That is, the person often experiences a philosophical shift in what he or she believes to be the most important things in life (Calhoun \& Tedeschi, 2006). Extrinsic priorities take a backseat to intrinsically important ones. Survivors also often report existential or spiritual growth often manifested in a greater sense of meaning in life, greater life satisfaction, and greater depth of spiritual experience (Caihoun \& Tedeschi, 2006). The impact on personal faith can be positive or negative, and some persons, experience existential crisis or a loss of faith as a result of their tragedy. This is especially true is the tragedy is considered senseless, or if the loss is great However, samples of survivors studied in the United States predominately report positive spiritual and religious change as a result to trauma (Calhoun \& Tedeschi, 2004).

Cognitive Engagement. Calhoun and Tedeschi (2006) have contended that rumination about a traumatic event or experience is necessary and even crucial for 
growth to occur. They have labeled this process as cognirive engagement and suggest that the level of posttraumatic growth reported by trauma sufferers tends to be directly correlated with the degree to which they have ruminated about the stressful event (Calhoun \& Tedeschi, 2006). Cognitive engagement involves the mental processes of meaning making, memory, cognitive restructuring (schema revision), and future . planning. For growth to occur, they suggest, the person must cognitively acknowledge and accept the reality of the trauma, and then regroup their mental resources to cope with that reality. Survivors move from thoughts of "How could this happen?" or "Why did this happen?" to "This has happened, how do I deal with it?" Several studies (e.g., Cozzolino, Staples, Meyers, \& Samboceti, 2004; Epstein, 1991) have found that the higher the level of threat and stress associated with the traumatic event, the more likely people are to report higher levels of growth, which has been found across several types of traumas (Calhoun \& Tedeschi, 2006). However, there is a point of diminishing return at which psychological coping capacity is exceeded. Therefore, more trauma does not necessarily lead to more growth in all people; the more severe a trauma, the more likely it will be to foster growth, as long as cognitive resources are not overwhelmed. Cognitive engagement, and hence posttraumatic growth, has several potential positive aspects. Traumas can be thought of as earthquakes that shake the assumptive foundations of cognitive schemas. Once these schemas are retuilt, they are more resistant to future assault. Also, experiencing a trauma, and growing from within, tends to make subsequent traumatic assaults to the system more psychologically manageable. 


\section{Posttraumatic Growth Following Cancer}

As has been described, receiving a cancer diagnosis can meet the criteria of a traumatic experience. Common symptoms of psychological trauma are often involved in dealing with cancer, inciuding fear, a sense of helplessness, imminent threat of death, challenges to core assumptions, uncertainly about the future, changes in interpersonal relationships, and invasive, painful medical treatments (Janoff-Bulman, 2004). Nevertheless, "some cancer patients report profound positive changes in themselves, their relationships, and other life domains after cancer"' (Stanton, Bower, \& Low, 2006, p. 138).

Despite her criticisms of posttraumatic growth research, Wortman (2004) has stated that mortality salience is the most central type of threat faced by cancer patients. Drawing on her own trauma research and personal work with cancer patients, she concedes that "across all the disasters, people who thought that they were going to die were most likely to report personal growth as a result of their experience" (Wortman, 2004, p. 85). Thus, it could be asserted that most diagnosed individuals report that they have experienced some type of psychological growth or have benefited in some way as a result of their experience with cancer. Furthermore, it is surprisingly common for people to say they are even glad they got cancer (Stanton, Bower, \& Low, 2006; Wortman, 2004). Positive growth has been reported by individuals diagnosed with various types of cancer including: breast, lung, colorectal, testis, melanoma, and prostate cancer. Current prevalence estimates of positive growth range from 60-95\% (Stanton, Bower, \& Low, 2006). 
The most common type of gowth reported by cancer patients is in the area of

interpersonal relationships More than a quarter of cancer patients surveyed across studies

report deeper, more meaningful relationshins with loved ones, as well as increased

compassion and aivareness of one's own importance to others (Stanton, Bower, \& Low, .2006). Greater appreciation for ife represents another common area of growth reported by cancer survivors. Between $60 \%$ and $85 \%$ of participants across studies reported a renewed outlook on life, changed priorities, and revised goals (Sears, Stanton, \& DanoffBurg, 2003; Stanton, Bower, \& Low, 2006). Notably for the present study, enhanced spirituality is also commonly reported (Cordova et al., 2001). Variables Affecting Growth Following Cancer

While positive benefits are reported by many cancer patients, such reports are not universal. Hence, it is important to consider the factors that make an individual more likely to report positive growth, that is, characteristics which distinguish those who experience growth from those who do not. Stanton, Bower, and Low (2006) compiled the results of over 50 studies in an effort to determine the common correlates of posttraumatic growth. Due to the correlational nature of this line of inquiry, studies yielding non-significant results (i.e., no relationship). Th1s is a limitation in that the reader may be mislead into interpreting non-significant results as significant ones. Also correlational data alone is insufficient to justify causation. However, non-significant studies have been included because they may also be useful in understanding to what variables posttraumatic growth is not correlated. All of the included studies that found significant results did so at the $p<.05$ level. This review of the research findings yielded factors that fell into the following categories: sociodemographic variables, characteristics 
of the stressor, personality attributes, social context variables, coping processes, and indicators of physical heaith.

Sociodemographic variables. No clear relationship was found between . socioeconomic status (SES) and positive growth. One explanation for this is the . incongruent measures of SES used by the various studies included. Researchers in three studies found a significant positive correlation between minority status and growth (Bower et al., 2005; Tomich \& Helgeson, 2004; Urcuyo et al., 2005), but several of the studies investigating ethnicity yielded a non-significant result. In terms of age, several . researchers found that younger patients tended to report more growth, but most of the studies in this category yielded non-significant results. To date, no research has found a significant relationship between gender and growth. Therefore, SES, minority status, age, and gender have not be shown to be correlated with reports of posttraumatic growth following cancer.

Characteristics of the stressor. There is strong evidence to suggest that the greater the perceived threat to life the more likely participants are to report posttraumatic growth. Positive, though less consistent correlations have been found between disease severity and psychological growth. Only one study found a significant relationship between reported growth and a more positive prognosis (Collins, Taylor, \& Skokan, 1990). The connection between how much time had passed since the cancer diagnosis and when : . growth was reportedly experienced was inconclusive. Also, the type of treatment received, and the type of surgery employed, did not have a significant impact on reports of growth. Overall, this means that the more life threatening a person perceived their cancer experience to be, the more likely they were to report psychological growth, 
whereas variables such as the passage of time and type of treatment employed did not appear to influence reports of growth.

Personality attributes. The personality variables of optimism, higher self-esteem, and reward responsiveness were ail found to be positively correlated with positive growth (Schulz \& Mohamed, 2004; Urcuyo et al,, 2005). Those who tend tó have a general expectancy for positive outcomes are more likely to report having grown from their experiences. Indeed, many cancer studies report at least a modest relationship between posttraumatic growth and the dispositional factors of optimism, hope, and extraversion (Affleck \& Tennen, 1996). Conversely, neuroticism and threat sensitivity have not been found to be significant correlates (Urcuyo et al., 2005). Thus, positive personal psychological resources have been positively connected to reports of posttraumatic growth.

Social context variables. A significant relationship between psychosocial factors and levels of reported posttraumatic growth has also been found..Cordova et al. (2001) found that the participants who talked with others about their experience with compassionate others were significantly more likely to report posttraumatic growth $(r=$ $.25, p<.05$ ). Also, merely being married does not in and of itself appear to foster posttraumatic growth, but persons with supportive partners have shown increased levels of growth (Schulz \& Mohamed, 2004; Weiss, 2004). Posttraumatic growth was also positively related to sustained contact with another person who had experienced such growth, and with the active seeking of social support as a strategy for dealing with cancer (Stanton, Bower, \& Low, 2006; Weiss, 2004). 
Coping processes. Persons engaging in the following coping strategies reported significantly more positive growth than those who did not: (a) problem-focused coping, (b) positive reappraisal, (c) acceptance, support seeking, (d) a more emotional coping approach, (e) more escape/avoidance coping, (f) less substance abuse, (g) religious coping, and (h) positive mood (Sears, Stanton, Danoff-Burg, 2003; Shultz \& Mohamed, 2004; Thornton, Perez, Meyerowitz, 2005;Urcuyo et al., 2005). It is relevant to note that, for each study yielding significant results for the above coping styles, there exists an accompanying study that yielded no significant result for that particular variable. This casts doubt on the strength of these findings. In coping research, a distinction is often drawn between coping processes of approaching versus avoiding the stressor. Overall, posttraumatic growth has been significantly connected to approach-oriented coping strategies, such as active acceptance. Also, longitudinal studies have shown that supportseeking is strongly related to growth, as people who are emotionally expressive and actively seek social support to cope are more likely to report growth (Stanton, Bower, \& Low, 2006). Moreover, increased grcwth has been reported by patients who see their illness as a challenge, that is, those who display a "fighting spirit" (Bower et al., 2005)

Physical health. Researchers investigating the relationship between physical health and posttraumatic growth distinguish between quality of life variables and more objective measures of physiological functioning. Overall there is only limited evidence that posttraumatic growth is associated with measures of one's general quality of life such as the Adjustment to Illness Scale or the Sickness Impact Profile (Stanton, Bower, \& Low, 2006). Although limited, there is evidence to suggest that posttraumatic growth is associated with more biologically oriented variables such as reduced serum cortisol 
(Cruess ct al., 2000), incirased levels of immune funcioning (McGregor et al., 2004), and less physical pain (Kat? et al., 2001). More research is needed to determine the direct relationship between posttraumatic growth and physical health before, during, and after the cancer experience.

In summary, immukable correlates of posttraumatic growth have yet to be described, and the existing literature on the subject is cften inconclusive and sometimes contradictory. The only correlates with substantial empirical evidence are those of social support and high perceived threat of the stressor. The equivocal nature of the findings of posttraumatic growth research following cancer should come as no surprise due to the nature of what is being studied. As Janoff-Bulman (2004) has noted:

The long-term legacy of trauma involves both losses and gains...the survivor can focus on one or the other, but both are ever present In the aftermath of trauma, survivers experience disillusionment and appreciation, unpredictability and preparedness, and vulnerability and strength (p. 34).

The Role of Spirituality in Posttraumatic Growth

Researchers have shown that many people experience growth following trauma, but some investigations are beginning to focus on the specific role of religion and spirituality in posttraumatic growth. Current research indicates that religious beliefs may be an important component of growth through traumatic experiences (Shaw, Joseph, \& Linley, 2005). For example, Schuster et al. (2001) conducted a survey immediately following the terror attacks of September 11, 2001 and found that $90 \%$ of those surveyed used their religious faith to cope. Evidence also exists to suggest that spirituality is sometimes experienced as helpful to people who are coping and recovering from 
traurnatic events (e.g., Emmons, Colby, \& Kaiser, 1998; Siegel \& Scrimshaw, 2000). Also, religious and spiritual beliefs have been shown to develop following a trauma, as has been reported in case studies of persons surviving war, illness, major disasters, and the Holocaust (Shaw, Joseph, \& Linley, 2005):

A wide range of responses to trauma, including both positive and negative aspects of religious and spiritual coping have been identified. Some benefits to using religion and spirituality to cope may include enhanced meaning/existential awareness, increased social support, and having a structured belief system (Shaw, Joseph, \& Linley, 2005; Vis \& Boynton, 2008). Also, spiritual coping may aid the reappraisal of a stressful event as less of a problem and more of a challenge, and may provide a fortified cognitive framework through which one may cope (Shaw, Joseph, \& Linley, 2005). Trauma may also provide the opportunity for those coping spiritually to experience an increased sense of personal meaning, as well as an enhanced and more highly developed spiritual life (Park, Edmondson, Fenster, \& Blank, 2008).

Negative harmful components of religious and spiritual coping have also been identified. For some, the experience of a traumatic event presents a strong challenge to their faith. Indeed, trauma, by definition, challenges presuppositions and spiritual assumptions. Persons may feel forsaken by God and experience bitterness, anger, or anxiety in their relationship with God (Shaw, Joseph, \& Linley, 2005). Moreover, some people report experiencing increased cynicism and decreased religious commitment following trauma (Shaw, Joseph, \& Linley, 2005).

Shaw, Joseph, and Linley (2005) reviewed the spirituality and posttraumatic growth literature and found consistent evidence for the importance of religion and 
spirituality to the process of posttrautnatic growth Qualitative research has revealed idiographic evidence of this, as religious coping has been reported to catalyze growth in abused women (Fallot, 1997), those experiencing a significant loss (Emmons, Colby, \& Kaiser, 1998), an ethnicaily diverse sample of women who were HIV positive (Siegel \& Schrimshaw, 2000), and parents of murdered children (Parappully, Rosenbaum, van den Daele, \& Nzewi, 2002). Quantitative evidence for the importance of spiritual coping to posttraumatic growth also exists and shows that traumatic experiences can stimulate religious and spiritual beliefs and that such belief can aid psychological recovery as well as personal development and growth (Shaw, Joseph, \& Linley, 2005).

\section{Spiritual Growth Following Cancer}

Research has shown that posttraumatic growth often involves a component of spiritual growth, and most modern growth scholars include a spiritual growth component as part of their theories. Descriptions of spiritual growth are often folded into more broadly defined categories. For example, Janoff-Bulman's (2004) postulates that posttraumatic growth involves a rejuvenation of religious and spiritual experience as part of enhanced existential awareness. Additionally, Calhoun and Tedeschi's (2006) model of posttraumatic growth incorporates renewed spirituality as part of its changed philosophy of life domain, which also includes a reevaluation of priorities and an increased appreciation for life itself. The Posttraumatic Growth Inventory (Tedeschi \& Calhoun, 1996) includes spiritual change as a subscale.

Researchers in the health and oncological sciences are also beginning to recognize that spiritual development and growth can evolve from the cancer experience, but current research findings lack specificity and depth. To date, the preliminary attempts to research 
spiritual growth following cancer have not sought to investigate spirituality in its fullest sense, but rather have looked only at a few specific facets of spirituality such as meaning in life, well-being, and prayer (Meraviglia, 2004; Meraviglia, 2006). For example, Meraviglia (2004) surveyed 60 adults with lung cancer who were between 33 and 83 years of age. They were given the Life Attitude Profile, Adapted Prayer Scale, Index of Well- Being, and Symptorn Distress Scale. Both meaning in life and prayer were positively correlated with psychological well-being. Meraviglia (2006) also surveyed 84 women with breast cancer and found that meaning in life again showed a strong positive correlation with positive coping. These studies $s_{s}$ though small and containing several limitations (e.g., small sample size, not having been replicate), have shown that an increased sense of meaning in life is associated with higher more positive coping and psychological health. Most studies addressing the connection between coping with cancer and spirituality have yielded little more than abstract findings (Albaugh, 2003; Gall \& Cornblat, 2002; Halstead \& Hull, 2001). Still, there is recognition of a vague connection between the traumatic experience of having cancer and spiritual growth, but there is currently little understanding of: (a) how this spiritual growth occurs, (b) what domains of spirituality are affected, (c) what type of spiritual growth follows cancer, or (d) how mental health and medical professionals can seek to foster such growth.

\section{Purpose Statement}

The purpose of the present study was to investigate how having cancer has affected the spiritual growth of survivors across a multidimensional conceptualization of spirituality using the twelve domains of spirituality postulated by Tsang and McCullough (2003) and Hill (2005). The goal was to investigate the phenomenological or lived 
'experiences: of postraumatic sniritual growth of cancer survivors. The interview protocol and analysis method were usea to ans'Ner the following everarching research question: How does surviving cancer impact the spiritual growth of survivors? 


\section{CHAPTER II}

\section{METHODOLOGY}

\section{Philosophical Paradigm}

Since research is built upon certain foundational assumptions, it is important to explicate the philosophical paradigm on which the present study was based (Colaizzi, 1978). This study rested on a constructivist philosophical paradigm, which is a way of knowing that emphasizes subjective interpretation of reality and the importance of contextual factors. Constructivism shares many of its underlying principles with cognitive theory and has roots in the cognitive movement (Neimeyer, 1993). According to McGrath and Johnson (2003), constructivism rests upon five primary assumptions: (a) reality is a highly subjective construction, (b) interpretations of reality cannot be divorced from personal perceptions and experience, (c) experience is highly individual and variable, (d) interpretations of reality are subject to change over time, and (e) knowledge is contextually based.

\section{Phenomenology}

Phenomenology is a type of qualitative inquiry that seeks to describe the meaning individuals give their lived experiences (Creswell, 1998), and is based on the philosophy that observable, measurable, duplicable (i.e., quantitative) approaches to psychological inquiry are prone to missing, or even altogether eliminating, the most important phenomenon under study, namely human experience (Colaizzi, 1978). It is an approach concerned not with explanation and control, but with understanding and description, and is "a refusal to tell the phenomenon what it is, but a respectful listening to what the phenomenon speaks of itself"' (Colaizzi, 1978, p. 52). 
There is no "one" phenomenological method (Colaizzi, 1978), but most approaches fall under one of twe major methodological umbrellas: either hermeneutic or empirical. Hermeneutic phenomenological approaches are concerned with analyzing and understanding written narratives to understand and describe human experience (Hein \& Austin, 2001). The goal is to produce a rich, deep description of a phenomenon as it emerges within a text, which can be done by analyzing the life-texts, or written experience of participants, or by studying previously written historical or literary narratives. During this process, the researcher works to overcome personal assumptions to understand and describe the phenomenon itself, as viewed in context from the text's perspective. Since phenomenology seeks to explore and describe the unique meaning assigned to experience by persons who have lived through a common phenomenon, it is an appropriate fit for the present study. As the goal of the study is to explore the lived spiritual experience of cancer survivors, the phenomenological approach developed by Colaizzi (1978) was used because its focus is on understanding and describing the lived experience of participants. Also, Colaizzi provides a flexible step-by-step process for data analysis (described below) the result of which is a description of the essence of participants' experience in terms of both their common and unique experience. Researcher as Co-Constitutor of Experience

At the time of data collection, the primary investigator was a 28 -year-old, European-American male completing his doctoral training in counseling psychology at a large state university in the South. He has completed a course in qualitative methodology, has collaborated on several qualitative research projects, and is currently working as one 
of the primary student research assistants on a qualitative research grant. He has intermediate experience with coilecting, analyzing, and interpreting qualitative data. Researcher Biases and Assumptions

Reflexivity is a strategy of critical seif-refiection that seeks to enhance overall rigor by requiring the researcher to explicitly state his or her potential biases and assumptions that may contaminate the research process or conclusions (Johnson, 1997, p. 283; Lincoln \& Guba, 1985). Following are the assumptions of which the researcher was currently aware. He believed that traumatic experiences can have a profoundly positive impact on one's spiritual growth. He expected that participants would report a significant amount of spiritual growth following their cancer experience within almost every domain of spirituality. In particular, he expected the domains of spiritual support, spiritual development, spiritual coping, and spiritual experiences to show the most significant growth. The researcher's own experience of studying issues related to spiritual growth following Hurricane Katrina may influence the way he saw the data, as a previous investigation yielded results that indicated spiritual development in trauma survivors. Ongoing discussions with the dissertation chair served to help ensure greater objectivity.

\section{Participants}

Thirteen persons participated in this study, which is in keeping with the current standard for number of participants in qualitative studies for counseling psychology (Hill et al., 2005; Hill et al., 1997). The primary inclusion criteria for the study was that participants received a diagnosis of cancer of any kind at some time in their lives and have subsequently been declared cancer-free by a physician who had medical evidence of the participant's cancer-free status (e.g., scan or blood test). Also, participants had to 
report that they believed their life was in danger st sone point during their cancer experience to be included in the sudy. Paricipants were identified by using key

$\therefore$ informants from the Hattiesburg, Mississippi chapter of the American Cancer Society. Upon receipt ơ proof of İRB approval (see Appendix E), the director of the Hattiesburg chapter of the American Cancer Society gave the researcher's contact information to potential participants. $A$ snowball samplirg procedure was used to identify participants who met criteria by utilizing peer-ncminations. Once a participant was identified, he or she was asked to identify another likely participant and so on. Every effort was made to include participants who represented a diverse range of demographic characteristics including gender, age, socioeconomic status, sexual orientation, ethnicity, and religious affiliation. Key informants and participants were specifically asked to nominate for participation persons who woul: diversify the sample.

Demographic information

The sample included three men and ten women. Twelve participants identified their ethnicity as Caucasian and one identified as African American. Participants ranged in age frorn 44 to 73 years with a mean age of 61 years. The amount of time each participant reported having been cancer-free ranged from 1 year to 23 years with a mean of 8 years. Seven participants were married, three were divorced, two were single and one was widewed at the time of the interviews. Eight participants had been diagnosed with breast cancer, two with Leukemia, one with colon cancer, one with lung cancer, and one with fallopian tube/ovarian cancer. The length of time participants reported having cancer ranged from 6 months to 22 years with a mean of 5 years. In terms of treatments participants underwent, eleven participants received chemotherapy, five had a single 
mastectomy, four had radiation treatment, three had a double mastectomy, three had surgery to remove a cancerous tumor, ani one had a lumpectomy. Four participants reported that they had cancer more than one time, with one participant reporting having : had cancer four times. In terms of religious affiliation, five participants identified as ... Baptist, five as Methodist, ‘wo as Catholic, and one as Latter Day Saint (Mormon).

\section{Procedure}

\section{Focus Groups}

Qualitative focus groups involve gathering together a group of persons with specialized knowledge relevant to the research question with the goal of informing and . . enhancing a study's research methodology (Marshall \& Rossman, 2006). They rely on group process dynamics to produce insights that emerge due to group interactions (Morgan, 1997). Prior to conducting the individual interviews, the researcher led three mini-focus groups, the goal of which was to refine the interview protocol. One focus group consisted of two cancer survivors, one was comprised of two religious leaders, and the third involved two medical professionals treating cancer patients (e.g., an oncologist, or oncological nurse). Members of the focus groups were asked to review the current interview protocol and offer feedback. The results of the focus group were used to refine the interview protocol.

Both the cancer survivors and the religious leaders indicated that using the term. "cancer free" would likely be problematic since there is no standard definition available. These focus group participants also warned that having cancer can become part of a person's identity and one cancer survivor proposed that there may be a difference between being medically and psychologically "cancer free." In response to this feedback, 
the interview protocol was amendec to usc lariguage such as "after your cancer experience..." and "as a carcer survivor.. " Moreover, participants were simply asked about their cancer experience in general terms and the "cancer free" label was not rigidly applied. Participants made it clear that they were beyond the "remission" phase and that they had not been dealing with the effects of having cancer for a specific period of time. Those in the cancer survivor's focus group staied that participants would likely make a distinction between religion and spirituality, thus a clarifying prompt was added to the interview protocol stating, "Is there a difference for you between religion and spirituality? If so, describe that?" Those in the ministers' focus group expressed that growth may be different depending on how disruptive the cancer experience was in the life of the survivor. In response to this feedback, more prompts were incorporated into the interview protocol that sought to tap how much of a disruption cancer was in participants' life. For example, participants were asked what treatments they underwent and how this affected them at work, home, and church. The medical professionals did not have any specific suggestions for changing the interview protocol, but did say that cancer survivors seem to look to the medical profession to provide answers and healing in addition to God. Thus, when participants talked about interactions with medical professionals as they described their cancer experience, the interviewer prompted them to talk about that person's role in their experience and how he/she may have impacted their spirituality.

\section{Instruments}

Two instruments were used in this investigation; a demographic questionnaire (see Appendix B) and a semi-structured interview protocol (see Appendix C). The demographic questionnaire was designed to help the researcher collect demographic 
information about participants, ensured that participants are cancer survivors, determined if participants believed their life was in danger during their cancer experience, and asked if participants' memory of their expericnce is vivid enough to be described. The semistructured interview protocol was used as a guide for conducting interviews. Each participant was asked each question on the protocol and follow up questions and prompts were used to increase the clarity and depth of responses.

\section{Individual Interviews}

The revised semi-structured interview protocol was then used to conduct individual interviews with participants. Participants took part in one-on-one phone interviews that were audio tape-recorded using the semi-structured interview protocol found in Appendix C. Once potential participants were identified, they were emailed an informed consent and asked to electronically sign the informed consent. Once the consent form was e-mailed back to the researcher and received, an interview was scheduled and the participant was sent the demographic questionnaire and the interview questions via email to review before the interview. Prior to beginning the interview, confidentiality was explained and participants were told that there are no correct answers to the interview questions and they were encouraged to freely relate their unique perspective. Participants were also informed that interviews would be audio recorded and transcribed verbatim. A one paragraph summary of each participart's interview was constructed by the researcher and emailed to participants who were asked to communicate whether they believed the summary accurately captured what they were trying to communicate during their interview. All participants indicated that the summaries accurately captured their experience. One participant clarified the type of cancer she reported surviving was 
inaccurate in the summ wy ithis had resulted from a type graphical error.

Phenomenoicgical qualitaive methotology was used to analyze the transeripts for emergent themes surrounding spiritual growth. Results described participants' lived experience of posturaumatic spirituai growin and are comprised of clusterea, cross-case themes and relevant participant quotes.

\section{Analycis}

\section{Qualitative Methodology}

Qualitative methodology is as a type of scientific inquiry that uses "data that are verbal and visual rather than statistical," and emphasizes the qualities or essences of the phenomenon under study (Morrow \& Smith, 2000, p. 199). It is well-suited to the research questions of psychology and has also been widely used in the fields of (a) anthropology, (b) sociulogy, (c) nursing, (d) social work, (e) administration, (f) community services, (g) maniagemeni, $(\mathrm{h})$ education, and (i) medicine (Merriam, 2002; Morrow \& Smith, 2000). Qualiiative methods are scientifically useful because they: (a) can be used to investigate research questions ill-suited to quantitative methods, (b) provide rich descriptions of particularly complex or multidimensional phenomena, (c) give voice to traditionally marginalized groups, (d) can serve as an initial exploration toward the development of theories or quantitative measures, (e) can illuminate the

diverse perspectives and experiences of several persons experiensing a similar event, and (f) often have a clear connection to applied practice (Sofaer, 1999).

Qualitative investigations are comprised of several common features that distinguish them from other types of scientific inquiry. For example, Patton (1990) has outlined ten primary chatacteristics of qualitative research: (a) naturalistic inquiry, (b) 
inductive analysis, (c) holistic perspeciive, (d) qualitative data, (e) personal contact and insight, (f) dynamic systems, (g) unique case orientation, (h) context sensitivity, (i) empathic neutrality, (j) design flexibility. Psychologists' interest in qualitative . approaches has grown in the past several years as evidence by recent special issues of professionals journals devoted to qualitative methods (e.g., Journal of Counseling Psycholcgy). Also, there has been a recent upsurge in the use of mixed models by psychological researchers (Morrow, 2007) along with an increased appreciation for how qualitative methods can be used to bridge the science-practice gap and how such methods are congruent with the paradigms that characterize counseling psychology.

Phenomenological Data Analysis

The present study employed the empirical phenomenological data analysis approach developed by Colaizzi (1978), and further elucidated by Worthen and McNeill . (2002). The approach offers step-by-step procedures that are meant to be a flexible, general guideline. The present study incorporated the following data analysis steps: First, Obtaining a Sense of the Whole involves gaining an overall sense of what each participant is communicating, which is necessary due to the extreme importance of context and the researcher's ability to understand the lived experience of participants. Therefore, all transcripts were read at least twice in their entirety to develop this overall sense. Second, Identifying Relevant Meaning Units involves identifying significant statements or phrases relevant to the phenomenon under investigation (spiritual growth). The researcher extracted these units of meaning from the transcripts by underlining relevant quotes and phrases in the text. Third, Articulating the Meaning Units means translating the meaning of participant statements into terms that are relevant to the 
research question and which ate most congruent with the phenomenon under study. Formulation of the meaning of the extracted phrases in this way requires going "beyond what is given in the original data and at the same time staying with it" (Colaizzi, 1978, p. 59). For the present study, the researcher summarized each underlined meaning unit with a word or phrase that is relevant to spiritual growth, writing it in the margin of the transcript. Fourth, Cross-Case Analysis calls for comparing the articulated meaning units of each protocol with the meaning units derived from all of the other protocols and clustering together units that are similar. The researcher organized the formulated meanings into cross-protocol theme clusters attending to the frequency (or relative infrequency) with which themes emerged across protocols. Fifth, the researcher created a Description of the Essence of the Experience, which involves generating a holistic description of the participants' aggregated experience. Results are presented in the form of domain labels/descriptions, which include representative participant quotes.

\section{Enhancing Rigor and Trustworthiness}

Lincoln and Guba (1985) have outlined four standards by which qualitative inquiry may be judged: (a) credibility, (b) transferability, (c) dependability, and (d) confirmability. The present study will incorporate rigor-enhancing strategies that specifically address each of these criteria.

Credibility. Credibility refers to the degree to which the results of a study have merit and accurately represent the experienced reality of its participants (Devers, 1999; Lincoln \& Guba, 1985). The method of triangulation, which involves using several sources of information to confirm a conclusion and is most often achieved through a combination of interview, observations, and data analysis, was used to address this 
criterion (Johnson, 1997; Merriam, 2002). In this investigation triangulation was achieved by interviewing several, diverse participants who have survived a similar phenomenon (i.e., cancer), using an outside auditor, who examined the obtained results, and integrating the findings into existing theory. Second, negative case sampling, involves actively seeking evidence that disconfirms the findings of a study (Lincoln \& Guba, 1985). The strength of this approach is that it "rnakes it more difficult to ignore important information" (p. 284) and it increases the credibility of the obtained results (Johnson, 1997). The researcher actively sought participants who did not experience positive spiritual growth following his or her cancer experience for inclusion. Participants and informants were directly asked if they knew any such persons, but none were identified. However, the researcher did report domains of posttraumatic spiritual growth not supported by this investigation as a form of disconfirming evidence. Third, referential adequacy--the degree to which findings can be shown to have a direct link to the actual words of the participants (Lincoln \& Guba, 1985) was used. Interviews were audiorecorded and transcribed verbatim to satisfy this requirement. The researcher then compared the tapes with the transcripts for accuracy, making necessary changes if inconsistencies were present Member checking, which involves asking participants to confirm the accuracy of the researcher's interpretation of the data helps ensure that researchers have appropriately interpreted participants' words (Johnson, 1997; Merriam, 2002). Member checking took the form of a brief summary of the interview that was written by the researcher and emailed to participants. Participants were then asked to confirm that the summary indeed captured the essence of what they wanted to 
communicate. All partielaris ind onten that the summary was an aceeptable depiction of their communication.

Transferability. Transferability refers to the degree to which findings can be generalized or transferred to simiiar peopie, settings, and times found in the originai study (Lincoln \& Guba, 1985; Merram, 2002). The researcher bears the responsibility of identifying key characteristics of the particlpants and context with the goal of giving the reader enough information to determine possible transferability (Devers, 1999). This is most often accomplished through the use of tinick descriptions, which involves providing rich detail about participant characteristics, statements, and context. (Lincoln \& Guba, 1985). Thick descriptions were incorporated through the use of participants' actual words to augment and support the results that emerged, and by presenting detailed participant demographic characteristics in their entirety.

Dependability. Dependable irivestigations can be relied upon to accurately and impartially report the findings that emetged from the data (Devers, 1999; Lincoln \& Guba, 1985). Dependability audits involve asking an objective, outside third-party to review the findings and check for disruptions in dependability (Lincoln \& Guba, 1985). In the present study, an experienced qualitative researcher with experience in phenomenological qualitative methods served as an official outside auditor. The outside auditor is a professor of healtheare management at a large Southern university who has severed as a reviewer for the National Institutes of Health as an expert qualitative methodologist. After reviewing and working the data shared by the researchers, the outside auditor reported achieving similar findings and verified that the obtained results were perceived to be accurate and reflective of the data. This person was given access to 
the primary researcher's transcripts (both coded and non-coded), codes, and domains to affirm the logic and dependability of the results. This person analyzed a portion of the transcripts herself and obtained similar results as those of the primary researcher.

Moreover, the scrutiny of the five members of the dissertation committee added to the study's dependability.

Confirmability. Confirmability refers to the degree to which the results of the investigation can be objectively corroborated by the obtained data (Lincoln \& Guba, 1985). The external auditor was also asked to perform a confirmability audit to ensure that the conclusions and results obtained by the primary investigator are verifiable from the perspective of a dispassionate peer (Johnson, 1997). This was done at the same time the dependability audit was carried out, as the auditor simultaneously. attended to issues of dependability and confirmability. Confirmability will also be enhanced by the triangulation, member checking, and referential adequacy strategies previously described. Also, field notes were taken to further enhance the confirmability of findings and to further add to the audit trail (Merriam, 2002; see Appendix D). 


\section{CHAPTER III}

\section{RESULTS}

Participants reported believing that their spirituality was strengthened and deepened as a result of their cancer experience. 'These participants reported seeing themselves as stronger in their faith and more aware of the importance of spirituality in their lives. Overall, the spirituality of these participants reportedly grew in importance, complexity, depth, and salience as a result of their experience with cancer. More specifically, participants reported posttraurnatic growth across eight of the twelve domains of spirituality postulated by Tsang and McCullough (2003) and Hill (2005). There were no noticeable differences in participant responses based on any demographic variables (e.g. age, diagnosis, etc). While not all participants experienced growth in the domains reported, no participant described an experience that contradicted the reported growth of any other participant. No pre-set number of participants reporting growth was used to determine the viability of a domain. but all cross-case domains reported were identified by at least four participants.

\section{Increased God Locus of Control}

Eleven of the thirteen participants reported that their spiritual growth was enhanced through acceptance of their circumstances and fully surrendering control of their lives to God. They asserted their belief that God was in charge of their circumstances (e.g., whether they lived or died, the effectiveness of treatments, etc.) and that neither internal nor external factors were superseding this control. Participants believed that this surrendering of control represented an increased level of spirituality in their lives and that it was a crucial component of the optimism and peace they 
experienced. In addition. these participants reported experiencing an increased trust in and dependence upon God as a result of having cancer. They described accessing this increased faith as a way to cope with their situation, a process which reportedly fostered both participants" spirituality and positive coping. As one participant noted, "I could not have made it through all of that without my faith." Conversely, one participant pointed to her cancer experience itself as the impetuous for increasing her faith and acceptance, "It. [cancer] makes you know that you have to exercise your faith to accept the things you can't change."

Nine participants specifically talked about accepting that God is in control of their lives and that they are not. They stated that, at some point during their bout with cancer, they came to believe that only God could help them overcome their circumstances. As one participant expressed, "Ultimately I have no control over this. I can turn it over to God and let him carry it." They talked about putting their lives in God's hands and reported that surrendering control to God made their cancer experience less emotionally distressing. One participant explained, "Whenever you figure out that you're not in control it just makes things go a lot smoother." Surrendering control of one's life to God was described as fostering participants' faith. Participants viewed surrender as a significant component of their spirituality and a catalyst of their spiritual growth. One participant explicitly linked acceptance of his cancer diagnosis with his spirituality saying, "Acceptance is the key to cancer. It is the key to religion, to spirituality," while another acknowledged, "Putting him [God] in charge of what I did instead of myself was probably how I grew the most." Participants also reported increased acceptance of the possibility of death and "how temporary this life is." In addition, participants reported 
reconciling their mortality with their setidulal belicts, as anghlighted by a participant who sairl "I was in that acceptance mode of "its $n$ if iny healing does not take place until heaven, that's fine."'

Two participants recalied the specitic moment they decided to surrender control of their struation to God. For these two participants, there was a defining moment of surrencier that, for them, was crucia! in the precess of coping with their diagnosis. One participant talked about getting on her harnds and knees in her bedroom and saying "God I need you and I can't do it without you." Another said,

I walked out in my front yard, looked up, my hands and arms to the sky and I said, 'Lord this is too big for me to handle by myself, so here I am. If you'll get me through it I'll do what you tell me to do.' I put my faith in the divine healing power. Prayer and faith is the strongest part of coping with cancer.

Though this could be could be interpreted as the participant seeking to bargain with God she described this experience more in the context of peaceful surrender than of making a deal with God.

Ten paricicipants reported an increased reliance on God as a result of their experience with cancer. This enhanced trust in God reportedly endured through their cancer experience as well as after it. Through their illness, these participants reported trusting God to give them strength, healing, courage, and the ability to endure their circumstances. While describing her thoughts during the approximate midpoint of her cancer experience, one participant said, "I was bald, I was swollen, I thought I was going to die. I had to just rest in the assurance that God was going to give me the strength to get through.". Several participants reported trusting that God was handling their medical 
condition. Two of these participants talked about trusting God over and above their doctors. It was salient for them to be "able to have enough faith to believe that God could work it out even when the doctors couldn't."

In addition to increased dependience on God through the experience, participants also pointed to an improved ability to rely on God post-experience. One participant described developing the ability to worry less about her life and that her cancer experience proved to her that God was trustworthy. She said,

I know I can trust him [God] because of what I went through. My trust deepened so much during that time. I don't worry like I used to. When something comes up I really can say, 'Ok God, I'm going to trust you with this.' I've learned to trust that he's a big God and he can take care of things for me and defend me instead of me taking things in my own hands and trying to fix all these problems. I think I just trust him more and let go of them [problems] more.

Overall, eleven participants reported an increase in their ability to trust God and surrender control of their lives to a divine power. Participants reported that, through these expressions of spirituality, they became more accepting their circumstances and believed the psychological and emotional distress caused by enduring cancer was ameliorated. Increase in Divine Peace

Eight participants reported experiencing an increased sense of divine peace, at some point during their cancer experience. Though participants reported experiencing a fear of death and the unknown as a result of having cancer, these participants reported that God eventually helped them overcome these fears and credited God for this sense of peace. They described feeling a profound, inward sense of serenity or well-being that , 
they had not experienced prior to having cancer and thai served to help them cope with the reality of their medicai condition. The experience of a sense of divine peace despite initial terror is typified in one participant's description:

I think the battie in your mind is probably the hardest battle when you are in cancer treatment because you are attacked in all directions to be fearful and to give up. I know that it was God battling that for me. All I had to do was depend on him [God] to make me not be afraid.

This peace was also explained as having a divine cause. "If God's plan for my life is that I'm gonna die from breast cancer, then I accept that. It's like a calmness and a strength that I can't explain." This internal sense of well-being was directly connected by participants to their spirituality. As another participant noted, "I had such a peace that came from my faith and my belief." After describing an extremely fearful night in the hospital following her initial diagnosis, one participant expressed feeling a sense of calm the next morning, which she believes came from God:

The next day whenever I woke up, I just felt this feeling of peace had come over me. I just felt like however things turned out that it was going to be ok. I was at peace. I know that the peace that I had is not something I could have done on my own because I was totally devastated. I just started feeling good about it; feeling like whatever happens is okay.

Three participants expressed feeling surprised at the peace they experienced, especially considering the traumatic, challenging nature of their circumstances. One participant expressed that she had not endured a traumatic experience prior to having cancer and "it was a surprise that I did have such a peace from God about everything." 
They also reported believing that God was helping then in multiple and unexpected ways and that their spirituality was positively impacted as a result of feeling at peace. Consider one participant's narrative:

Where is this peace and this evenness in my life coming from? Well, it is coming from God. It was coming from him ministering to me and helping me each day to cope with it [cancer] and live through it and heal, not just physically, but to heal spiritually as well.

In addition, another participant noted that she feels a sense of peace about her future in relation to cancer. She reported fearing death in general before she was diagnosed, but that she does not now fear dying even of cancer. She described this divine peace as seen extending beyond the cancer experience itself into her post-cancer experience. This participant explained:

I have made the choice not to live in fear of it [cancer] coming back. I believe in my heart that it may eventually be what I die of, but we are all going to die of something. People that know me will just say that it seems there is such a calmness now. I don't have the fear. I leave it in God's hands and I do not have any of that fear and anxiety when I do.

Over half of participants reported experiencing a sense of peace and calm that they had not felt before being diagnosed with cancer. For many participants, this peace was experienced despite a time of feeling extreme fear about the reality of having cancer and at the prospect of dying. The cancer experience was viewed as the reason this peace was so intensely felt and divine peace was believed to be experienced despite and because of having been diagnosed with cancer. 
Enhanced I'rayer Experisnce

Nine particinants teporter that having cancer increased the quaiity and depth of their prayers; though tinis dia not necessarily translate into increased frequency or duration of prayers. Particinants reported that they now experience more vigor in their prayers, and that that prayier is more imporiant to them than it was prior to their cancer experience Ore participant related, "it increased the soncentration and intensity and energy that I put into them [prayers]." Participants also expressed that prayer had become less ritualized and more integrated into the lived experience of their daily lives. As one participant noted, "They [prayers] are just more conversational now. Instead of having a separate prayer time, it is just more a part of everything I do throughout the day. Just that awareness of God in every situation." Another participant noted that, for her, prayer was not as formal an activity as it had been before having cancer. She reported an increased awareness of God's presence in her life and that she more often talks to God spontaneously. She stated, "It is not so much a formal thing, but it is an informal prayer life... I am just more aware of 1 . It is an ongoing thing." Another participant reported conceptualizing prayer as a less complex activity after having cancer. She described her belief that God is aiways listening and that she does not always have to know exactly what to say to have a positive prayer experience. She explained, "Sometimes I can just hold up my hands and say 'God I need you' and it doesn't have to be this long prayer."

Further, participants reported feeling a deeper connection with God in their prayers as result of their cancer experience. They described this connection in terms of an increased sense of intimacy in communication with the divine and an emotional closeness with God that emerged during their cancer experience and continues to persist following 
their struggle with cancer. As one participant noted, "The best way I can describe it is just more of a personal relationship [with God] now." Another participant stated, "It brought me closer [to God]. It seemed like having that cancer put me sure enough in his arms. It's just like it's a closer bond now." One participant spoke of often incorporating into her prayer time, both during and after her cancer experience, a visual image of herself in God's lap. She expressed feeling more emotionally connected to God when she had cancer than she did at any other time in her life. She said,

I probably became more teachable during that time. And because I was so needy I was more willing to listen. I would just picture myself literally sitting in his [God's] lap with my head against him, and it wasn't like that before. It just made me seek Him in a different way because of how he literally met every one of those needs. It has really made me look at him like a daddy.

Participants talked about avoiding compartmentalizing their spirituality to the degree he did prior to having cancer. That is, spirituality is now experienced as pervading all domains of participants' lives versus being viewed as a distinctly separate component of their experience. One participant spoke of sensing God's presence in all aspects of his life and feeling hè now has a more intimate relationship with God. He stated, "He [God] is such a part of everything I do every day. There is no part of my life that I do not feel that I am experiencing him."

On the whole, participants reported that the quality of their prayers increased as a result of their experience with cancer. They described a more profound relational closeness with God as well as an ability to pray with increased comfort, spontaneity, and 
energy. For these participants, prayer became less ritualistic and more of an ongoing activity pervading their daily experierce.

Increased Spiritual Support

Six participants reported receiving increased spiritual support from their family and friends as well as people in their faith community during their cancer experience. Participants expressed that the spiritual support they received exceeded any they had received in the past and that it came from many people. As one participant explained, "Everyone I. was aware of rendered increased spiritual support to me during this experience... family and friends. Certainly in terms of prayer, expressing their faith, expressing encouragement, fasting." In addition, participants reported receiving increased unsolicited spiritual support from family and friends and that people showed this support through being physically present with the participant. One participant highlighted her experience as she lay in a hospital bed:

I feel like they.[family/friends] really did rally and were very supportive. I had people that were just lifting me up in every possible way. I had a steady stream of people coming through my [hospital] room and I had all kinds of people pray with me in my room. It was just an experience that I wouldn't take anything for.

Several participants expressed surprise at the unexpectedly strong spiritual support they received from friends, family, and even people they had never met. For example, two participants described receiving supportive communications such as cards, letters, emails, and phone calls from people they did not know personally, even from persons in other countries. As one participant noted, 
When I was diagnosed with cancer, I was just overwhelmed with the support I got. I had people writing me and teling me that I was in their prayers and I received letters and phone calls from people I didn`t really know.

Participants also described experiencing strong spiritual support within their faith communities. They talked of being spontaneously prayed for by people with whom they had previously had only a casual relationship. Clergy and church members reportedly visited participants both at home and in the hospital. As one participant related, "I had lots of people from my church visiting me and oh my gosh, the cards I got." Participants described being placed on the prayer lists of churches they had never attended.

People in participants' faith community also provided a combination of spiritual and practical support. One participant reported that several church members would come to her house while she was taking chemotherapy, do housework for her, and pray with her. Another participant was approached by two ladies in her church who were cancer survivors and who offered her medical advice and prayer. Participants also reportedly having spiritual experiences with fellow congregants that they did not have prior to their cancer experience. For example, one participant related that, though prayer with supportive physical contact was rare in her faith community, "People would just come up behind me [at church] and touch me and pray for me." Another participant related that, though prayer was often done quietly in her church, more congregants prayed for her out loud. One participant expressed amazement at the spiritual support she received from her minister. She stated that each time she had a doctor's appointment “....my pastor would be sitting there waiting when I walked in the door [of the cancer center]." 
Thus, participants reponter experiencing increased spiritual support from family, friends, and people in their faith communitis. Particinants also expressed surprise at the spiritual support they received and reporte: having novel spiritual experiences with others that were thought to nave been catalyzed by their cancer experience. Heightened Sense of Divine Purpose

Eight participants repurted feeling an increased sense of a divine purpose for their having had cancer. While no participants reported that they believed God purposely gave them cancer, several reported a belief that God used their experience for a spiritual purpose. According to participants, this heightened sense of divine purpose provided them with meaning and a spiritual explanation for their difficulties with cancer. It also gave them an increased sense of overall purpose and meaning to their lives in their postcancer experience. For example, one participant expressed her belief that she has been commissioned to work for God following her cancer experience saying, "I know he [God] has a plan for my life and that I have work to do."

While all eight of these participanits specificaily mentioned divine purpose, two were able to describe this sense of purpose in only vague terms. While they were unable to articulate what specific divine purpose their cancer may have had, these two participants made definitive statements affirming their belief in a divine purpose for their cancer experience saying, "... everything happens for a reason and my cancer was for a reason," and "...he [God] allowed this to happen and there was a purpose in it." After admitting that she does not know the purpose for her cancer experience or for her life after cancer, one participant was adamant in her belief that there is a purpose, though 
unknown. She said, "Everyday it is like 'God there is a reason I am here' I do not know the reason, but there is no doubi in my mind that there was a purpose.".

Six participants were able to point to specific potential divine purposes for their cancer experience, including: to bring them closer to God, to bring others closer to God, and to make them able to provide support for others. Their experiences were seen as . means which God used to foster their life purpose and to bring about the plan he has for them:

There's no doubt that everything I've been through, God has brought it to this incredible plan for me. And I will gladly have gone through this and whatever else I need to go through to bring about his purpose for me.

Two participants stated that the divine purpose for their cancer experience was to increase their devotion and obedience to their spiritual beliefs. One of these participants believed that God was using her cancer experience to increase her commitment to her faith. She said, "I think, through this experience, he [God] was calling me into a deeper relationship with him and to step it up a level." The other participant stated, after explaining that he had lived many years rejecting his spirituality and engaging in behaviors that are inconsistent with his religious values, "God gave me a chance to do something about my life." This person believed that God gave him a second chance to live a spiritual life in adherence to his beliefs.

Two participants believed the divine purpose for their cancer experience was to bring others closer to God. These participants said they now "have a great testimony" of God's goodness they could share in an effort to persuade others to pursue spirituality. They expressed their belief that their cancer experience produced increased courage and 
ability to serve as an exampie to others of how God bririgs people through difficult circumstances. They also reported a desire to teil others of the importance of maintaining faith in God during trying times. "If my talking about it could bring even just one person closer to finding their faith in God, that might be God's only reasen for allowing me to have it. And that's a good enough reason."

Two participants viewed their cancer experience as being used by God to enhance their ability to support others who are struggling. As one participant explained:

Maybe it [the reason for having cancer] was to be able to be there for other people, to listen to them, to tell them that I am a cancer survivor, to make them feel a little more at ease about what they are going through.

One participant expressed her belief that the purpose of her cancer experience was to increase her empathy for and ability to positively respond to others who are facing cancer. This participant, a high school English teacher, described providing support for students facing a potential cancer diagnosis, saying, "I had several students, young ladies, who came and they had a lump in their breast and I would listen and sometimes I would share and sometimes we would just talk. This might be my purpose."

Six participants expressed appreciation for the lessons having cancer taught them about themselves and God. They reported learning that their faith was stronger than they expected and that they were pleased with their ability to remain spiritual and courageous through their experience. As one participant expressed, "I have a lot more faith than I ever knew I did." These participants also described learning more about God's characteristics, specifically God's love, trustworthiness, and kindness. One participant 
stated that, "He [God] taught ine thai I could totally trust him with my life, literally." Another participant noted,

I learned more about his [God's] love. I felt his love. I learned more about his understanding, his iong suffering, his patience, his forgiveness, his caring and nurturing, his wisdom, his understanding and knowledge. I learned more in-depth about each of these than ever before.

It appeared important to participants to communicate their belief in a significant, divine reason for their cancer experience, as well as their sense of enhanced personal meaning and purpose in their lives as a direct result of having cancer.

\section{Increased Evangelism}

Four participants reported increased willingness and opportunity to verbally communicate their spirituality to others following their cancer experience. These participants expressed that they were more motivated to talk to others about their spiritual beliefs after having cancer than they were before. As one participant expressed, "Before I had cancer, I didn't share God with others like I should. I give my testimony a lot more often than I used to." In addition, participants reported that they experienced an increased eagerness to communicate their faith to more people. For example, one participant talked about being more open to letting others know her after she had cancer and telling more people about her faith in God. She stated, "It [having cancer] has given me the desire to accept others into my life and to reach out to others."

Participants expressed that, after having cancer, they were bolder, more outgoing, and increasingly assertive in sharing with others what they believed God had done for 
them. These participants viewer their expenence vith calicer as increasing their courage to publiciy express their spirituality. As one participant related:

I never would have been one that would have gone up front and prayed on the aiter or talked to anyiody about my feelings. That Sunday whenever I got to go home [from the hospital], I went to church and stood in front of the church and told everybody what had happened and about the miracle and their prayers and what it had done for me. I would have never done that before. I have completely changed. I just feei like anybody that will listen I'll tell about my story and I've probably told it a million times.

Another participant reported being afraid to tell other people about her spiritual beliefs before she had cancer. Now that she is a survivor, she reported less trepidation around teiling others about her faith. She reiated that being a survivor gave her increased courage to tell others her beliefs about God because people seemed more willing to listen to her talk about her faith because she had survived cancer. She said,

I was always afraid to talk to anybody about my faith. But I've found most people don't mind hearing it. I was supposed to have died but I didn't and I feel God had a part in that. Most people take that kind of seriously.

Participants also asserted their belief that God was providing them opportunities to communicate their faith through telling others about their experience with cancer. Several participants reported being asked to speak to both religious and nonreligious groups of people about their experience, both of which were open to hearing about participants' spirituality As one participant noted, “... this [having cancer] has just opened a lot of doors and gave me more opportunities to share God with people." One 
participant reported feeling that he was obeying God when he spoke about his faith to a group of people. Another participant stated, "I think God is bringing me slowly but surely in contact with more people that I can listen to or share with or just be a friend to, and just show love, Christian love, Christ-like iove."

The cancer experience was believed, by these four participants, to have developed within them increased courage to disclose spiritual information about themselves to others. Moreover, as a cancer survivor, these participants were provided with an increased number of opportunities to share their cancer experience, which for them included sharing information about their spirituality.

Enhanced Spirituality of Family and Friends

Seven participants expressed believing that their experience with cancer had a positive effect on the spirituality of their family and friends. While two participants reported having specific conversations with their loved ones about spiritual growth, all seven of these participants reported that they observed behaviors that they interpreted as spiritual growth. Participants observed these behaviors in the context of their families and their faith communities. In terms of spiritual growth within the family, participants reported increased spiritual practices of family members such as family prayer. One participant observed, "As a family, we prayed more together." Another participant related that she feels her family takes their spirituality more seriously as a result of her having survived cancer. She said, "I think there has been a renewed sense of the importance of faith in my family:" In addition, participants reported that their family experienced an increased sense of commitment to their faith. Not only did spirituality come to increased salience for these participants' families, but they reportedly experienced qualitative 
growth in their spirituality. As one participant stated, "To some extent every member of my family experienced a deepened spirituality ard a heightened commitment to the Lord."

Four participants reported that their children, in particular, experienced personal spiritual growth as a direct result of their bout with cancer. These participants expressed that their children's spirituality' grew through watching how they themselves remained firmly adherent to their spirituality despite having cancer. As one participant stated, "Not only did cancer re-affirm my farth, it helped my children have faith." One participant reported that his son had previously rejected the family's faith tradition and had a difficult time reconciling how a loving God could allow his father to have cancer. He conveyed that his son's faith was enhanced despite this, saying, "My son was able to build up a deeper reservoir of faith that allowed him to come to terms with this and to become at peace with it." Another participant noted an increased willingness of her children to participate in their faith community, saying, "One of my sons went to youth and they had a prayer vigil for me the night they found out."

One participant stated her belief that her cancer experience was the impetus for increased spiritual activity among people in her faith community. She reported that a friend in her church had been considering starting a ministry for people who were sick. This ministry would focus on meeting the practical needs of the ill (e.g., cleaning house, washing clothes, cooking meals) until they felt better. This participant stated, "When she found out I had cancer, immediately she put that ministry in place."

In summary, participants reported that they believed the spirituality of their friends and family was positively impacted as a result of their cancer experience. 
Participants talked of increased spiritual practices within the family as well as a belief that their family members' faith was broadened and deepened. Moreover, participants observed increased spiritual practices among people in their faith communities, with some people increasing their wiilingness to pray in pubiic and others engaging in new ministries.

\section{No Growth Domains}

Spiritual growth was not reported across the domains of religious or spiritual history, religious or spiritual beliefs and values, religious or spiritual techniques for regulating and reconciling relationships. The religious and spiritual history of participants was unaffected as having cancer did not change or grow the degree to which a person's family heritage involved religion and spirituality. Since it stands to reason that a current experience cannot affect a person's history, the participants' reflections or potential changes in narratives were listened for. Overall, there was no report from participants that their cancer experience affected the meaning they gave their spiritual heritage. Further, all participants reported that having cancer did not change any specific religious or spiritual beliefs. For example, when asked if having cancer affected her belief system, one participant said, "No, I do not think so. Not at all...I just faced it [cancer]. This was just another chapter in my life."

Finally, participants reported that there was no growth in their ability to use their spirituality to reconcile conflict in relationships. Mosi participants reported that they did not experience conflict during their cancer experience. One participant stated, "I just didn't have any difficulties [in relationships] that I remember." One participant reported that her spirituality sometimes causes conflict when she talks to people who disagree or 
. . do not want to talk about spirtun! matters. She said, "You can"t preach spirituality to somebody, but you can try. Scmetimes they cion't want to hear it and it rnakes conflicts." However, this participant did not link this to having cancer, but instead related that this is an experience she has had prior to cancer. Participants did report being more patient in their relationships after haviting cancer and that they do not take minor irritations in their relationships less seriously. As one participart noted:

I have learned what is important and what's really not. There are some things that just aren't worth having conflict over. I have a lot more patience. I do think I've learned to just be a little bit more laid back about most things.

Despite this, only one participant linked the experienced of increased relational patience to spirituality. This participant reported that the enhanced spirituality he experienced indirectly impacted his ability to handle relational conflict. He said, "Heightened spirituality brings greater understanding, greater patience, deepened love, and all of those things have a significant effecr on how you handle conflict with others." Overall, participants credited their increased ability to handle conflict in relationships to their experience of almost dying rather than to increased spirituality. 


\section{CHAPTER IV}

\section{DISCUSSION}

The present study asked participants about their perceptions of their spiritual growth across 12 domains of spirituality described by the Hierarchical Model proposed by Tsang and McCullough (2003) and Hill (2005). Growth was reported in eight of these domains: general religiousness or spirituality, religious or spiritual development, .. religious or spiritual social participation, religious or spiritual private practices, religious or spiritual support, religious or spiritual coping, religion or spirituality as motivating forces, religious or spiritual experiences, and religious or spiritual commitment. Two novel domains of growth emerged from participants' narratives: evangelism and enhanced spirituality of family/friends. These experiences have not yet been reported in the literature. Growth was not endorsed by participants in the following three domains of spirituality outlined in the model: religious or spiritual commitment, religious or spiritual history, religious or spiritual beliefs and values, and religious or spiritual techniques for regulating and reconciling relationships.

All of the participants in this study reported that their spirituality grew or was enhanced as a result of their experience with cancer, some even reporting they had experienced "profound" and "remarkable" spiritual growth. Spirituality has been found to be a significant component of posttraumatic growth (Calhoun \& Tedeschi, 2006; Mattis, 2002; Shaw, Joseph, Linley, 2005; Vis \& Boynton, 2008), and research findings suggest that some people report spiritual growth following a traumatic experience such as debilitating illness (Calhoun \& Tedeschi, 2006; Davis, Wohl, \& Verberg, 2007; Halstead, \& Hull, 2001; Park et al., 2008; Park \& Ai, 2006). In addition, participants reported 
experiencing spiritual growth across several domains of spirituality. Participants reported an increased sense of depth and meaning in their spiritual experiences and a heightened awareness of the presence of God in their day-to-day lives. Also, participants believed that the spirituality of their family and friends was rostered as a result of their experience with cancer and they reported an increased urge to communicate about their faith with others through telling their cancer story and what they believe God did for them through the experience. Overali, relatively littie is currently known about the impact of traumatic experiences on spiritual growth and the facets of spirituality most impacted by those experiences.

Areas of Growth Reported

Participants experienced an increased sense that God was in control of their lives and circumstances during their cancer experience. They attributed their positive health outcomes to a divine will and power and believed that God intervened in their circumstances, helping them cope and avoid dying. Consistent with this finding, a sense of control over one's life has been associated with positive psychological well-being and healthy aging (Krause, 2006). People who are highly religious score higher on a measure of God locus of control, and attempts have been made to correlate God locus of control with health outcomes (Shapiro, Schwarz, \& Austin 1997; Wallston et al., 1999). The results of the present study appear to be in contradiction to findings of other studies that have suggested that God locus of control may have deleterious effects on well-being. For example, Wallston and colleagues (1999) found that, in a sample of people with rheumatoid arthritis and systemic sclerosis, increased God locus of control was associated with poorer adjustment. In addition, a sample of persons participating in Alcoholics 
Anonymous who had more God lacus of control beliefs about their drinking were found to remain sober for a shorter time than those who have an internal locus of control (Murray, Malcarne, \& Goggin, 2003).

In contrast, for the participants in the present study, giving control to God appeared to serve to ameliorate the psychological and emotional distress caused by enduring cancer as well as to boost their ability to engage their faith to cope. Research has shown that people may experience increased anxiety and overall distress following a traumatic experience if they believe that no one is in control of life circumstances (Koenig, 2006). Wong-McDonald and Gorsuch (2000) found that giving control of one's life to God and accepting that one cannot control his or her circumstances was associated with spiritual well-being. Moreover, Cole (2005) found that individuals who believed God was in control of their illness experienced lower levels of depression and physical pain. It is possible that participants in the current study reported positive effects of giving God control of their circumstances because they were highly religious and spiritual or due to having experienced ar end to their traumatic experience with cancer. Less spiritual people who continue to struggle with the medical treatments associated with cancer may benefit more from an internal versus a God locus of control.

Despite being diagnosed with cancer and being facing with their mortality, participants in the present study reported an increased sense of divine peace and internal . well-being throughout.their cancer experience. Participants reported that their peace came from an enhanced emotional and psychological connection with God. This coincides with Gall and Cornblat's (2002) finding that cancer survivors who felt a strong divine presence in their life were more likely to seek increased spirituality. Feeling "at peace" 
has been strongly correlater with errotiona! ant spritual well-being in one study of cancer patients (Steinhauser et al, 20,06). However, there is a dearth of literature investigating cancer patients' expenence गÏ peacs.

It is possible that the peace reported by participants in the present study fostered their hope, which could have boosted both their sense of well-being and spiritual growth. If participants' hope stemmed from their spirituality, it is likely that they sought to foster that spiritualıty, which may have led to increased divine peace. Rustoen and Wiklund (2000) found that all of the recently diagnosed cancer patients they surveyed reported feeling either hopeful or moderately hopeful. Felder (2004) also found that hope was strongly correlated to positive coping in cancer patients regardless of gender, age, marital status, education, or site of malignancy, and even in patients who were aware of the advanced stage of their cancer.

Participants reported an increase in the quality and intensity of their prayers both throughout and following their cancer experience, Holt and colleagues (2009) reported that cancer patients often report increased frequency of prayer, which was not supported by the finding of the present study, in which participants reported an increased depth but not increased frequency in their prayers. According to several studies, prayer is one of the most commonly used religious coping strategies to deal with stressful life events (e.g., Gall \& Cornblat, 2002; Halstead \& Fernsler, 1994; Pargament; 1097; Spilka, Hood, Hunsberger, \& Gorsuch, 2003). Meraviglia (2002) reported that prayer can be a valuable coping resource that can mitigate the negative psychological impact of cancer. As was the case in this study, persons with cancer frequently use prayer to cope with the distress caused by their symptoms and the procedures they often undergo, and spirituality has 
been identified to be importani component of the coping of long-term cancer survivors (Halstead \& Fernsler, 1994; Meraviglia, 2006; Stanton, Bower, \& Low, 2006). Consistent with the findings of the present study, Levine and colleagues (2009) found that breast cancer survivors who prayed were more likely to report that having cancer had a positive impact on their lives. Private spiritual practices including prayer have been shown to predict survival among people experiencing long-term disability (Helm et al., 2000). Prayer has been associated with psychological growth in cancer patients, with increased prayer intensity positively correlated with well-being (Meraviglia, 2006; Meraviglia, 2004), resilience and existential hopefulness (Carson, 1993; Poloma \& Pendleton, 1991); and a sense of positive life meaning in cancer survivors (Gartner, Allen, \& Larson, 1991; King, 1990). Taylor and Outlaw's (2002) review of the literature on prayer as a coping strategy revealed that there is currently little empirical knowledge of the specific prayer experiences of cancer survivors, and that no studies had investigated survivors' prayer experiences with any depth from survivors' point of view.

Participants reported receiving increased spiritual support from the people in their lives during their experience with cancer and that this support led to spiritual growth. Consistent with this, increased religious social support has been reported by persons experiencing personal crises (Stone, Cross, Purvis, \& Young, 2003). Further, increased social support levels were found to be associated with higher levels of spirituality in patients diagnosed with HIV/AIDS (Somlai \& Heckman, 2000). Balboni and colleagues (2007), who surveyed 230 patients with advanced cancer, found that patients who perceived strong spiritual support from their family, friends, and medical providers reported increased quality of life. Moreover, Hughes and colleagues (2004) found that 
religious and spiritual social support served as a bufier against anxiety in patients with coronary heart disease. Among persons forcib!y removed by the government from the Gaza strip, high religious support was predictive of lower PTSD symptoms (Oren \& Possick, 2009).

While participants in the present study did not report increased frequency of church attendance as a result of their cancer experience, they did report attending church with approximately the same frequency before, during, and after their experience. These findings contradict previous research suggesting that cancer patients sometimes, upon diagnosis, increase the frequency with which they attend church (Moschella, Pressman, Pressman, \& Weissman, 1997). This could be explained by the fact that most of the participants in the present study reported being highly spiritual prior to their cancer diagnosis and most reported being involved in a faith community from the time they were children.

The sample in the present study was comprised predominantly of women, which may also partially explain why spiritual support emerged as a salient theme, as the positive benefit of religious involvement on health has been consistently shown to be greater for women (Ellison \& George, 1994; McCullough et al., 2000; Mirola, 1999). In addition, the spiritual support received by participants in the present study likely contributed to their ability to make positive meaning out of their experience and thus come to view having cancer as a growth experience. Social support has been shown to facilitate meaning-making (Lepore, Ragan, \& Jones, 2000; Park \& Ai, 2006).

Participants described an increased desire and ability to discover a divine purpose for their experience with cancer. Participants were motivated to understand and articulate 
the potential reason they had cancer and what God expected them to do with their lives now that they were cancer-free. Spiritualiiy has been described a meaning-making activity "whereby individuals grapp!e with the purpose and value of human life in general, and of their own lives in particular" (Cook \& Kelly, 1998, p. 3). Researchers have argued that spirituality and meaning making are inseparable aspects of cognitive reevaluation following an existentially laden stressor 'Calhoun \& Tedeschi, 2004; JanoffBulman, 2004; Park, 2005). Participants in the present study reported utilizing increased spirituality to facilitate their meaning-making process, which is also consistent with existing literature. Research has shown that survivors of traumatic events attempt to understand the meaning of the stressor (Lepore \& Revenson, 2006; Park, 2005), and that religious and spiritual meaning-making often plays a crucial role in the process of coping with negative life events (Calhoun \& Tedeschi, 2006; Pargament, 1997). Such spiritual meaning-making has been found helpful in trauma recovery and to reduce the symptoms of PTSD resulting from traumatic experiences endured by refugees (Brune et al., 2002), rape survivors (Boeschen et al., 2001), survivors of the September 11 terror attacks (Silberman, 2005), and survivors of childhood cancer (Parry \& Chesler, 2005). Further, meaning-making has been found to be extremely common among cancer survivors and has been identified as an important factor in their psychological growth (Park, Edmondson, Fenster, \& Blank, 2008; Greenstein \& Breitbart, 2000; Schroevers, Ranchor, \& Sanderman, 2004; Tomich \& Helgeson, 2002). Cancer patients in particular have been found to consistently construct meaning for their suffering (Kappeli, 2000), and Park and colleagues (2008) found that cancer survivors' who actively engaged in meaning-making reported better overall adjustment. The results of the present study provide further 
support for research findings showing the positive impact of spiritual meaning-making on psychological growth (Calhoun \& Tudeschi, 2004).

Novel Growth Areas Reported

Farticipants reported that, both while they nad cancer and after, they experienced an increased desire to tell other penple about their spiritual beliefs with the goal of bringing othere closer to God. In fact, one of the most salient reasons participants in the current study believed they had cancer was so they could communicate their cancer story and faith to others. Self-disclosure has been shown to have overall benefits and has been associated with increased coping behaviors, psychological resilience, enhanced work performance, and better physical health (Pennebaker, 1997). Spiritual self-disclosure has been related to increased relationship satisfaction, less verbal aggression, and more functional communication patterns between adolescents and their mothers (Brelsford \& Mahoney, 2008). Henderson and colleagues (2002) for example, found that disease disclosure was positively related to stress-related growth and optimism. Participants in the present siudy reportedly experienced more than a simple desire to disclose information; the goal of their disclosures was to persuade others to adopt their beliefs. Despite the preliminary research on spiritual self-disclosure, the evangelistic theme that emerged in the present study is novel and has received very little attention in the literature.

Participants reported that the spirituality of their family and friends was enhanced as a result of their having had cancer. This is a novel finding that emerged from this study. Researchers have acknowledged, however, that religious and spiritual experience does not occur in a vacuum, but that there is an important psychosocial component of 
these experiences (Pargament, 1997). Some researchers have sought to measure vicarious posttraumatic growth with one study finding that both cancer patients and their partners experienced increased posttraumatic growth during several points in time following the diagnosis (Manne et al., 2004). Also, Weiss (2004) found that husbands of cancer survivors reported posttraumatic growth three years after their wives were diagnosed. Despite these preliminary attempts to describe vicarious growth, the spiritual components of vicarious growth have yet to be researched.

The significant people in the lives of participants in the present study reportedly served a therapeutic function, and it is possible that friends and family members who * support survivors may themselves experience some positive personal benefit from assuming the helper role. Research findings that show therapists who work with trauma victims may experience positive vicarious benefits such as increased appreciation for : human resilience, enhanced empathy, and deepened spirituality (Arnold, Calhoun, Tedeschi, \& Cann, 2005; Brady, Guy, Poelstra, \& Brokaw, 1999; Schauben \& Frazier, 1995). Overall, there is a dearth of research investigating vicarious posttraumatic growth and vicarious posttraumatic spiritual growth has not been addressed in the literature. As Arnold and colleagues have noted: "Very little is known about the nature, prevalence, impact, and process of psychological growth following vicarious brushes with traumathe phenomenen of vicarious posttraumatic growth" (p. 243).

No Growth Domains Reported

Spiritual growth was not reported across the domains of religious or spiritual history, religious or spiritual beliefs and values, religious or spiritual techniques for regulating and reconciling relationships. It is reasonable to assume that a person's 
spiritual heritage is unaffected by a traumatic event, as it is impossible to alter past events. It is currently unclear exactly how religious and spiritual beliefs are impacted by traumatic experiences. Some stıdies have found that trauma may have a negative effect on beliefs (e.g., Finkelhor, Hotaling, i.ewis, \& Smith, 1989; McLaughlin, 1994; Rossetti, 1995) while others have found it to have a positive effect (e.g., Carmil \& Breznitz, 1991; Valentine \& Feinauer, 1903). One recent study found that people who reported more severe symptoms of PTSD were also more likely to report less religiosity following their traumatic experience (Falsetti, Resick, \& Davis, 2003). Participants in the current study differed significantly from participants in Falsetti and colleagues' study in that they were highly religious and spiritual prior to their cancer experience. The effect of trauma on spiritual beliefs may be mediated by the level of commitment one feels to those beliefs prior to the trauma. In terms of relational conflict, trauma has been shown to produce conflict in interpersonal relationships including relationships with partners, friends, coworkers, family, and friends (Mills \& Turnbull, 2004). Despite this, cancer survivors often report less relational conflict and increased intimacy and support in their interpersonal interactions (Stanton, Bower, \& Low, 2006). Consistent with this, participants in the present study reported receiving strong spiritual and social support from the people in their lives. These participants did not experience significant conflict in their relationships and so did not report growth in using their spirituality to cope with conflict.

\section{Limitations}

The present study is subject to the limitations of qualitative research. Results cannot be generalized to a larger population of cancer survivors as they seek only to 
describe the individual lived experierce of the thirteen participants and have only transferability: Further, results are only explanatory in nature and cannot be used to control or predict the spiritual growth of cancer survivors. Researcher bias is another potential limitation, though refiexivity steps were taken to minimize effects. Finaliy, results cannot be used to determine causality, as they seek only to describe the phenomenological experience of pcsttraumatic spiritual growth.

Although several rigor-enhancing methodological techniques were used to ensure the accuracy of the results, there are some limitations to this study. First, all of the participants identified themselves as belonging to the Christian faith. Though they represented several groups within Christianity, the bulk of participants were of relatively homogeneous groups, with 10 identifying as Baptist, 10 Methodist, two Catholic, and one Latter Day Saint. Therefore, results may not exiend to members other groups within Christianity or other world religions, and to those who consider themselves to be atheistic or agnostic. Also, the sample was only comprised of persons in middle to late adulthood and results may have been impacted by generational or age variables.

A second limitation is that over half of the participants (eight) were breast cancer survivors. It is possible that breast cancer survivors have a different experience than persons who survive other types of cancer. Moreover, the sample represented a gender disparity (ten women and three men). There may be gender differences in the experience . of post-traumatic spiritual growth and the results of this study were more strongly influenced by the experiences of female participants as there were simply more of them in the sample. Also, all but one participant identified as Caucasian (one identified as African American). People of various racial, ethnic, or cultural heritages may have 
- differing expecisnces of spiritulity and spiritual growth, and different themes may have emerged if the, sample had been more heterogeneous.

- Selt-selection is another limitation of this study. People drawn to participate in the study seemed to be those with an active interest in communicating positively about their spirituality and comfortabie verisatizing their belıefs. Ail participants considered themselves highly spiritua! people, and different results would likely have emerged from a sample of survivors who censider themselves less spiritual or altogether unspiritual. Though efforts were made to include participants who felt their spirituality was negatively impacted by their cancer experience, such persons were not identified. This could be a result of the snowball sampling procedure (i.e., those willing to participate did not know anyone whose spirituality was negatively impacted) or that people who had a negative experience were simply not interested in participating. Also, bias might have been introduced through the use of the contact at the American Cancer Society. This person may have been motivated to ccntact only people who have had a positive experience following cancer. Aiso, people affiliated with the Cancer Society may be more socially involved and positive about their cancer experience than people who are not affiliated.

In addition, all participants reported being highly spiritual before their cancer experience, and it is possible that necple who are spiritual pre-experience are more likely to use their spirituality to cope or attend to spiritual issues than persons who do not consider themselves spiritual. Alsc, no baseline level of spirituality prior to participants' cancer experience was obtained. Different results may have emerged if the sample 
included people who either were not spiritual prior to their cancer experience and now are, or who were not spiritual before and became so during their experience with cancer.

Finally, in keeping with the values of Counseling Psychology and the positive psychology movement, the present study was strength-based and growth-focused. Such studies are subject to the general criticisms of all growth research, namely: an overly . optimistic presentation of results that betrays a bias toward positive changes, and disclosure bias whereby participants are less willing to disclose negative experiences. To mitigate these potential biases, the present study presented areas in which growth was not reported, and participants were specifically asked if they had any negative spiritual experiences associated with their cancer experience.

\section{Implications and Future Research}

Spirituality is a multi-faceted construct and relatively little is known about its specific domains. Previous research has not asked participants to consider one hypothesized spiritual domain at a time. The present study further elucidated the domains of spirituality by specifically asking participants to consider their experience of each individual domain. The result was confirmation of several domains offered in the literature, while at the same time bringing attention to two additional novel themes of spirituality. Thus, this study may help further clarify and expand definitions of spirituality.

The current study showed that people who survive cancer may report spiritual growth across several domains of spirituality. Participants in the present study described spiritual growth along several domains of spirituality. Future research could focus on further describing and explaining the growth experienced across these domains. In 
addition, researchers could conduct larger scale quantitative studies that seek to describe the frequency, duration, depth, and breadth of this growth as well as the demographic characteristics of those who experience growth.

Since spirituality can be a significant source of coping, it may be helpful for medical and mental health professionals to understand how spirituality may be positively impacted by the experience of having cancer and how spiritual growth can be facilitated. if this will aid coping. For example, researchers could further investigate each individual domain of spirituality to discover the environmental, interpersonal, and intrapersonal variables that foster its growth. Further, survivors could be asked what components of their spirituality were most and least useful to them through their cancer experience and what they did to engage their spirituality to cope.

The findings of this study suggest that people close to those enduring cancer may also experience spiritual growth. This vicarious spiritual growth has not yet been researched and it may be useful for mental health professionals and clergy to better understand how a persorı's traumatic experience may positively impact others in a family, group, or community. Researchers could further study this phenomenon through quantitative methods seeking to correlate the spiritual well-being of survivors with that of the significant people in their lives. Qualitative methods could be used to explore the variables and experiences associated with vicarious posttraumatic spiritual growth and its progression.

The results of the present study suggest the spiritual domains within which growth may occur, but further investigation is needed into the variables in a person's experience that may foster or hinder this growth and into how posttraumatic spiritual growth occurs. 
Future research could focus on how spiritual growth occurs through traumatic experiences and how spirituality could be used to potentially enhance the positive coping of people who are experiencing traumatic events. Researchers could use qualitative methods to further describe the process and experience of spiritual growth. To begin, a more broad qualitative study could be performed which included a larger and more diverse set of participants in terms of religious affiliation, demographic variables, and . type of cancer survived, etc. Beyond that, the novel themes of evangelism and vicarious posttraumatic spiritual growth could be explored qualitatively through studies investigating survivors' experience of spiritual self-disclosure following their traumatic experience as well as the spiritual growth of persons with loved ones who have endured trauma. Further, mixed model designs could be used to develop a measure of posttraumatic spiritual grewth by further bringing to salience important domains of growth. For example, cancer survivors could be given a preliminary measure of posttraumatic spiritual growth, which could be factor analyzed and tested for reliability and validity. Participants could then be interviewed in-depth as to their experience growth, which could further illuminate important domains of growth or serve to refine domains. Quantitative methods could then be used to investigate the corollaries and predictors of growth and to explore the relationship between spiritual growth and global mental and physical health outcomes. For example, the relationship between depression. and spiritual growth following cancer could be investigated. Also, spiritual growth could be used as a moderating or mediating variable. For example, a study could investigate how posttraumatic spiritual growth may mediate resilience and coping, anxiety, or existential well-being in both cancer patients and survivors. 
APPENDIX A

Informed Consent

\section{The University of Southern Mississippi}

\section{Authorization to Participate in Research Project}

Consent is hereby given to participate in the study titled: Positraumatic Spiritual Growth: A Phenomenological Investigation of Cancer Survivors

1. Purpose:

I understand that the purpose of this study is to explore is to explore the

experiences of cancer survivors in terms of and how having cancer has affected the spiritual growth of survivors.

\section{Description of Study:}

I understand that I will be participating in a one-on-one phone interview with a researcher. I understand that the interview will last approximately 40-90 minutes, and will be audio taped and transcribed verbatim The information collected from the interview will be examined and analyzed by the lead researcher. I understand that this interview does not incorporate any invasive procedures. I further understand that I will be sent a copy of the transcribed interview to verify that the researcher properly transcribed the interview.

\section{Bencfits:}

I understand that I will not receive any dirset benefits froni this study. Broader benefits of this investigation may include: (1) suggestions for improvement in the delivery of psychological services to persons dealing with cancer (2) implications for future research (3) a deeper understanding of how traumatic events affect a person's spiritual growth (4) reduction of feelings of isolation (5) the opportunity to give a voice to those marginalized by their cancer experience.

\section{Risks:}

Risks associated with this research are minimal. I understand that I may experience some discomfort as a result from thinking about, and discussing events and emotions related to my experiences. In addition, I understand that I can stop participating in the study at any time without any consequence. I understand that I will be able to contact the principle investigator Ryan M. Denney, M.A. at any time throughout the study at (940)613-1755 or at: rdenney1980@yahoo.com, or his supervisor Jamie D. Aten, Ph.D. at (601) 266-6246 or Jamie.Aten@usm.edu.

\section{Confidentiality:}

I understand that, to protect my identity, my audio tape and resulting transcript will be assigned an identification letter and my name will not be used to identify 
my audio tap. All transcripts, tapes, data, and any other information collected in the interview will be contained in a locked and secure space. After this study is completed my audio taped interview will be erased. My responses will not have any identifying information, and the researcher will take every reasonable precaution to protect my confidentiality.

\section{Alternative Procedures:}

I understand that I may stop participating in this study at any time without consequence.

\section{Subjects Assurance:}

Whereas no assurance can be made concerning results that may be obtained (since results from investigational studies cannot be predicted) the researcher will take every precaution consistent with the best scientific practice. Participation is completely voluntary, and participants may withdraw from the study at without penalty or negative consequence. However, once the audiotapes have been transcribed and de-identified by being given an identification number, participants will no longer be able to remove their participation (their transcripts) from the study. Questions concerning the research should be directed to Ryan M. Denney, M.A. at (940) 613-1755 or at: rdenney1980@yahoo.com. This researcher is working under the supervision of Jamie D. Aten, Ph.D., who can be reached at (601) 266-6246 or Jamie.Aten@usm.edu.

This project and this consent form have been reviewed by the Institutional Review Board, which ensures that research projects involving human subjects follow federal regulations. Any questions or concerns about rights as a research subject should be directed to the Chair of the Institutional Review Board, The University of Southern Mississippi, 118 College Drive \#5147, Hattiesburg, MS 39406-0001, (601) 266-6820.

\section{Signatures:}

I understand that, by replying to this email, electronically typing my name to this consent, and scheduling a time to be interviewed, I am acknowledging that I have read and understand the above information and I am giving informed consent to participate.

To electronically sign your name to give consent, please type your full name in the box below: 
APPENDIX B

Dernographic Questionnaire

Code

Phone

Email Address

Age

Gender

Marital Status

Ethnicity (Caucasian, African American, Asian, Native American, etc.)

Religious Affiliation

Type of cancer you had

Length of time you had cancer

Length of time you have been deemed "cancer free" by a physician

There was a time during my cancer experience when I believed my life was.in danger.

$\square$ Yes $\quad \square$ No

My memory of my cancer experience is vivid enough that I could talk about what this experience was like for me.

$\square$ Yes $\square$ No

I consider my cancer experience to have been a traumatic event in my life.

Yes $\quad \square$ No 


\section{APPENDIX C \\ Interview Protocol}

1.. Will you please briefly describe your experience with cancer? (Prompt: How did you first discover you had cancer? What kind of treatments did you undergo?)

2. What is your personal definition of spirituality? (Prompt: Is there a difference for you between spirituality and religion?)

3. Overall, how do you feel your experience with cancer has affected your spirituality?

4. How might your cancer experience have affected your motivation or desire to be spiritual?

5. How might your cancer experience have affected your private spiritual practices such as prayer, meditation, etc. (e.g., quality, frequency, duration, intensity)?

6. How might your cancer experience have affected the way you experience spirituality in your day-to-day life?

7. How might your cancer experience have affected your level of commitment to your spiritual beliefs?

8. How might your cancer experience have changed or developed your spirituality?

9. How might your cancer experience have affected the spirituality of your family or close friends?

10. How might your cancer experience have affected your involvement in a religious or spiritual community (e.g., church, synagogue, etc.)?

11. How might your cancer experience have affected the spiritual support you received from others?

12. How might your cancer experience have affected how you handle conflict in relationships?

13. How might your cancer experience have affected the way you use spirituality to cope with difficult life circurnstances?

14. Was there any negative impact on your spirituality during your experience with cancer?

15. Is there anything we have not discussed, which you think will help me better understand how your cancer experience may have affected your spirituality? 


\section{APPENDIX D}

Field Note

Participant Code:

Date:

Gender:

Àge:

Length of Interview:

Type of Cancer:

Themes

Follow-up topics

Researcher Impressions 


\author{
APPENDIX E \\ Institutional Review Board Approval
}

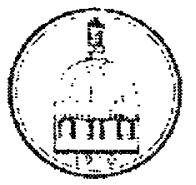

THE UNIVERSITY OF SOUTHERN-MISSISSIPPI

Instintutionas Rextew Boara

118 Collowe Drive 15147

Hatticsburg, MS 39460 ono1

Tel: 601.260 .0820

Fax: $(001.2605509$

wawn.umbirb

\title{
HUMAN SUBJECTS PROTECTION REVIEW COMMITTEE NOTICE OF COMMITTEE ACTION
}

The project has becn reviewed by The University of Southern Mississippi Human Subjects Protection Review Committe in accordance with Federal Drug Administration regulations (21 CFR 26, 111), Department of Health and Human Sevices (45 CFR Pant 46), and university guidelines to ensure adherence to the following criteria:

- The risks to subjects are minimized.

- The risks to subjects are reasonable in redation to the anticipated benefits.

- The selection a subjects is equitable.

- Infomed consent is adequate and appropriately documented.

- Where approprate, the research plan makes adequate provisions for montoning the data collected to ensure the safety of the subjacts.

- Where appropriate, there are adequate provisions to protect the privacy of subjects and to maintain the confidentiality of all data.

- Appropriate additional safeguards have been inchuded to profsct vunerable subjects.

- Any unanticipated, serious, or continumy problems encountered regarding risks to subjects must be reported immediately, but not later than 10 days following the eyent. This should be reported to the IRB Office via the "Adverse Effect Report Form".

- If approved, the maximum period of approval is limited to twelve months.

Projects that exoeed this period must submit an application for renewel or continuation.

PROTOCOE NUMBER: 27121001

PROJECT TITLE: Posttraumatic Spiritual Growth: A Phenomenological

Investigation of Cancer Survivors

PROPOSEO PROJECT DATES: $12 / 01 / 107$ to 08/31/09

PROJECT TYPE: Dissertation or Thosis

PRINCIPAL INVESTIGATORS: Ryan M. Donney

COLLEGEIDVISION: Collage of Education \& Psychology

DEPARTMENT: Counseling Psychology

FUNDING AGENCY: N/A

MSPRC COMMITTEE ACTION Expedited Review Approval

PERJOD OF APPROVAL: $12 / 10 / 07$ to $12 / 09 / 08$

starumer a thenmom

Lawrence A. Hosman, Ph.D. HSPRC Chair

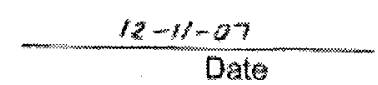




\section{REFERENCES}

Affleck, G., \& Tennen, H. (1996). Constructing benefits from adversity: Adaptational significance and dispositional underpinnings. Journal of Personality, 64, 899-922.

Ai, A.L., Cascio, T., Santangelo, L.K., \& Evans-Camball, T. (2005). Hope, meaning, and growth following 9-11. Journal of Interpersonal Violence, 20, 523-548.

Albaugh, J. (2003). Spirituality and life-threatening illness: A phenomenological study. Oncology Nursing Forum, 30, 593-598.

Allport, G.W. (1950). The individual and his religion: A psychological interpretation. New York: Henry Holt.

Allport, G.W., \& Ross, J.M. (1967). Personal religious orientation and prejudice. Journal of Personality and Social Psychology, 5, 432-443.

Altemeyer, B., \& Hunsberger, B. (1992). Authoritarianism, religious fundamentalism, quest, and prejudice. International Journal for the Psychology of Religion, 2, 113 133.

American Psychiatric Association. (2000). Diagnostic and statistical manual of mental disorder ( $4^{\text {th }}$ ed. text revision). Washington, DC: Author.

Arnold, D., Calhoun, L.G., Tedeschi, R., \& Cann, A. (2005). Vicarious posttraumatic growth in psychotherapy. Journal of Humanistic Psychology, 45, 239-263.

Balboni, T.A., Vanderwerker, L.C., Block, S.D., Paulk, M.E., Lathan, C.S., Peteet, J.R., \& Prigerson, H.G. (2007). Religiousness and spiritual support among advanced cancer patients and associations with end-of-life treatment preferences and quality of life. Journal of Clinical Oncology, 25, 555-560.

Batson, C.D., Schoenrade, P.A., \& Ventis, W.L. (1993). Religion and the individual. 
New York/Oxford: Oxford Universiiy Press.

Bearon, L.B., \& Koenig, H.G. (1990). Religious cognitions and uise of prayer in health and illness. Gerontologist, 30, 249-253.

Bjorck, J.P., \& Cohen, L.H. (1993). Coping with threats, losses, and challenges. Journal of Social and Clinical Psychology, 12, 36-72.

Boeschen, L.E., Koss, M.P., Figueredo, A.J., \& Coan, J.A. (2001). Experiential avoidance and post-traumatic stress disorder: A cognitive mediational model of rape recovery. Journal of Aggression, Maltreatment and Trauma, 4, 211-245.

Bolt, M. (1975). Purpose in life and religious orientation. Journal of Psychology and Theology, 3, 116-118.

Bower, J.E., Kemeny, M.E., Taylor, S.E., \& Fahey, J.L. (1998). Cognitive processing, discovery of meaning, CD4 decline, and AIDS-related mortality among bereaved HIV-seropositve men. Journal of Consulting and Clinical Psychology, $66,979-986$.

Bower, J.E., Meyerowitz, B.E., Desmond, K.A., Bernaards, C.A., Rowland, J.H., \& Ganz, P.A. (2005). Perceptions of positive meaning and vulnerability following breast cancer: Predictors and outcomes among long-term breast cancer survivors. Annals of Behavioral Medicine, 29, 236-245.

Bowker, L.H. (1988): Religious victims and their religious leaders: Services delivered to one thousand battered women by clergy. In A.L. Horton \& J.A. Williamson (Eds.), Abuse and religion: When praying isn't enough (pp. 229-234).Lexington, KY: Lexington Books.

Bradley, D.E. (1995). Religious involvement and social resources: Evidence from the 
data set: "Aniericaus" changing lives" Jowmal for the Scientific Study of Religion, 34, 259267.

· Brady, J. L., Guy, J. D., Poelstra, P. L., \& Brokaw, B. F. (1999). Vicarious traumatization, spirituality, and the treatment of sexual abuse survivors: A national survey of women psychetherapists, Prefessional Psychology: Research and Practice, 30, 386-393.

Brelsford, G.M., \& Mahoney, A. (2008). Spiritual disclosure between older adolescents and their mothers. Journal of Family Psychology, 22, 62-70.

Brune, M., Haasen, C., Krausz, M., Yagdiran, O., Bustos, E., \& Eisenman, E. (2002). Belief systems and coping factors for traumatized refugees: A pilot study. European Psychiatry, 17, 451-458.

Cadell, S., Regehr, C., \& Hemsworth, D. (2003). Factors contributing to posttraumatic growth: A proposed structural equation model. American Journal of Orthopsychiatry, 73, 279-287.

Calhoun, L.G., \& Tedeschi, R.G. (2004). The foundations of posttraumatic growth: New considerations. Psychological Inquiry, 15, 93-102.

Calhoun, L.G., \& Tedeschi, R.G. (2006). The foundations of posttraumatic growth: An expanded framework. In L.G. Calhoun \& R.G. Tedeschi (Eds.), Handbook of posttraumatic growth: Research and practice (pp. 3-23). Mahwah, NI: Erlbaum.

Carmil, D., \& Breznitz, S. (1991). Personal trauma and world view: Are extremely stressful experiences related to political attitudes, religious beliefs, and future orientation? Journal of Traumatic Stress, 4, 393-405.

Carroll, S. (1993). Spirituality and purpose in life in alcoholism recovery. Journal of 
Studies in Alcohol, 54, 297-301.

Carson, V.B. (1993). Prayer, meditation, exercise, and special diets: Behaviors of the hardy person with HIV/AIDS. Journal of the Association of Nurses in AIDS Care, $4,18-28$.

Cohen, S. (1988). Psychosocial models of the role of social support in the etiology of physical disease. Health Psychology, 7, 269-297.

Cohen, S., Doyle, W.J., Skoner, D.P., Rabin, B.S., \& Gwaltney, J.M. (1997). Social ties and susceptibility to the common cold: Journal of the American Medical Association, 277, 1940-1944.

Colaizzi, P.F. (1978). Psychological research as the phenomenologist views it. In R.S. Valle \& M. King (Eds.), Existential-phenomenological alternatives for psychology (pp. 48-71). New York: Oxford University Press.

Cole, B.S. (2005). Spiritually-focused psychotherapy for people diagnosed with cancer: A pilot outcome study. Mental Health, Religion, and Culture, 8, 217-226.

Collins, R.L., Taylor, S.E., \& Skokan, L.A. (1990). A better world or a shattered vision? Changes in perspectives following victimization. Social Cognition, 8, 263-285.

Cook, E. P., \& Kelly, V. A. (1998). Spirituality and counseling. Counseling and Human . Development, 30, 1-16.

Cordova, M.J., Cunningham, L.C., Carlson, C.R., \& Andrykowski, M.A. (2001). Posttraumatic growth following breast cancer: A controlled comparison study. Healthy Psychology, 20, 176-185.

Cozzolino, P.J., Staples, A.D., Meyers, L.S., \& Samboceti, J. (2004). Greet, death, and values: From terror management to transcendence management theory. 
Personality and Social Psychology Bulletin, 30, 278-292.

Creswell, J.W. (1998). Qualitative inquiry and research design: Choosing among the five traditions. Thousand Oaks, CA: Sage Publications.

Cruess, D.G., Antoni, M.H., McGregor, B.A., Kiïbourn, K.M., Boyers, A.E., Alferi, S.M., et al. (2000). Cognitive-behavioral stress management reduces serum cortisol by enhancing benefit finding among women being treated for early stage breast cancer. Psychosomatic Medicine, 62, 304-308.

Davis, G.D., Wohl, M.J.A., Verberg, N. (2007). Profiles of posttraumatic growth following an unjust loss. Death Studies, 31, 693-712.

Devers, K.J. (1999). How will we know "good" qualitative research when we see it? Beginning the dialogue in health services research. Health Services Research, 34, $1153-1188$.

Dienstbier, R.A. (1989). Arousal and physiological toughness: Implications for mental and physical health. Psychological Review, 96, 84-100.

Ellis, A. (1980). Psychotherapy and atheistic values: A response to A.E. Bergin's "Psychotherapy and religious values." Journal of Consulting and Clinical Psychology, 48, 635-639.

Ellison, C.G., Boardman, J.D., Williams, D.R., \& Jackson, J.S. (2001). Religious involvement, stress; and mental health: Findings from the 1995 Detroit area study. Sacial Forces, 80, 215-249.

Ellison, C.G., \& George, L.K. (1994). Religious involvement, social ties, and social support in a southeastern community. Journal for the Scientific Study of Religion, $33,46-61$. 
Ellison, C.G., \& Taylor, R.J. (1996). Turning to prayer: Social and situational antecedents of religious coping among African Americans. Review of Religious Research, 38, $111-131$.

Emmions, R.A., Colby, P.Mi., \& Kaiser, H.A. (1998). When losses lead to gains: Personal goals and the recovery of meaning. In The human quest for meaning: $A$ handbook of psychological research and clinical applications, P.T. Wong, \& P.S. Fry (Eds.) pp. 163-178. Mahwah, NJ: Lawrence Erlbaum.

Epstein, S. (1991). The self-concept, the traumatic neurosis, and the structure of personality. In D.J. Ozer, J.M. Healy, and A.J. Stewart (Eds.), Perspectives in personality, Vol 3 (pp. 63-98). London: Jessica Kingsley.

Erickson, S.J., \& Steiner, H. (2001). Trauma and personality correlates in long term pediatric cancer survivors. Child Psychiatry and Human Development, 31, 195213.

Exline, J.J., \& Rose, E. (2005). Religious and spiritual struggles. In Handbook of the psychology of religion and spirituality, R.F. Paloutzian \& C.L. Park (Eds.), pp. 315-330. New York, NY: Guilford Press.

Exline, J.J., Yali, A.M., \& Sanderson, W.C. (2000). Guilt, discord, and alienation: The role of religious strain in depression and suicidality. Journal of Clinical Psychology, 56, 1481-1496.

Fallot, R.D. (1997). Spirituality in trauma recover. In Sexual abuse in the lives of women diagnosed with serious mental illness, M. Harris, \& C. Landis (Eds.). Amsterdam, ‘ Netherlands: Harwood Academic Publishers, pp. 337-355. 
Palsett, S.A. Resick, P.A., \& Vavis, I.K. (2003). Changes in religious beliefs following trauma. Journal of 'Traumatic Stress, 16, 391-398.

Felder; B. (2004). Hope and coping in patients with cancer diagnoses. Cancer Nursing, $27,320-324$

Ferraro, K.F., \& Koch, J.R. (1994). Religion and heaith among black and white adults: Examining social support and consolation. Iournal for the Scientific Study of Religion, $33,362-375$.

Fetzer Institute/National Institute on Aging Working Group. (1999, October). Multidimensional measurement of religiousness/spirituality for use in health research: A report of the Fetzer Institute/National Institute on Aging Working Group. Kalamazoo, MI: John E. Fetzer Institute.

Finkelhor, D.: Hotaling, (G. T., Lewis, I. A, \& Smith, C. (1989). Sexual abuse and its relationship to later sexual satisfaction, marital status, religion, and attitudes. Journal of Interpersonal Violence, 4, 379-399.

Frankl, V.E. (1969). The will to meaning. New York: New American Library.

Freud, S. (1927). Future of an illusion. In J. Strachey (ed. and trans.), Standard Edition of the Complete Psychological Works of Sigmund Freud. London: Hogarth Press.

Gall, T.L., \& Cornblat. M.W. (2002). Breast cancer survivors give voice: a qualitative analysis of spiritual factors in long-term adjustment. Psycho-Oncology, 11, 524 535.

Gallup, G.H. (2003). Public gives organized religion its lowest rating. Gallup Poll Tuesday Briefing, pp. 1-2.

Gallup, G. H., \& Lindsay, D. (1999). Surveying the religious land-scape: Trends in U.S. 
beliefs. Harrisburg, PA: Morehouse.

Gartner, J., Allen, G.D., \& Larson, D.B. (1991). Religious commitment and mental health: A review of the empirical literature. Journal of Psychology and Theology, 19, 6-25.

Gorsuch, R.L., \& Butler, M. (1976). Initial drug abuse: A review of predisposing social psychological factors. Psychological Bulletin, 83, 120-137.

Greenstein, M., \& Breitbart, W. (2000). Cancer and the experience of meaning: A group psychotherapy program for people with cancer. American Journal of Psychotherapy, 54, 486-500.

Gurin, G., Veroff, J., \& Feld, S. (1960). Americans view their mental health: $A$ nationwide interview survey. New York: Basic Books.

Halstead, M.T., \& Fernsler, J.I. (1994). Coping strategies of long-term cancer survivors. Cancer Nursing, 17, 94-100.

Halstead, M.T., \& Hull, M. (2001). Struggling with paradoxes: The process of spiritual development in women with cancer. Oncology Nursing Forum, 28(10), 1534 1544.

Hantman, S., \& Solomon, Z. (2007). Recurrent trauma: Holocaust survivors cope with aging and cancer. Social Psychiatry and Psychiatric Epidemiology, 42, 396-402.

Hathaway, W.L. (1999). Impairment in religious functioning as a clinically significant issue for diagnosis. In W.L. Hathaway (Chair), Clinically Significant Religious Impairment: Diagnostic and Practice Issues. Symposium conducted at the annual. meeting of the American Psychological Association, Boston.

Hein, S.F. \& Austin, W.J. (2001). Empirical and hermeneutic approaches to 
phenomenological research in psychology: A comparison. Psychological Methods, 6, 3-17.

Helm, H.M., Hays, J.C., Flint, E.P., Koenig, H.G., \& Blazer, D.G. (2000). Does private - religious activity prolong survival? A six-year follow-up study of 3,851 older adults. Journals of Gerontology Series A-Biological Sciences \& Medical Sciences 55, 400405.

Henderson, B.N, Davison, K.P., Pennebaker, J.W., Gatchel, R.J., \& Baum, A. (2002). Disease disclosure patterns among breast cancer patients. Psychology and Health, $17,51-62$

Hill, C.E., Thompson, B.J., Hess, S.A., Knox, S., Williams, E.N., \& Ladany, N. (2005). Consensual qualitative research: An update. Journal of Counseling Psychology, $52,196-205$

Hill, C.E., Thompson, B.J., \& Williams, E.N. (1997). A guide to conducting consensual qualitative research. The Counseling Psychologist, 25, 517-572.

Hiil, P.C. (2005). Measurement in the psychology of religion and spirituaiity: Current status and evaluation. In R.F. Paloutzian \& C.L. Park (Eds.), Handbook of the psychology of religion and spirituality (pp. 43-61). New York: Guilford Press.

Hill, P.C., \& Pargament, K. (2003). Advances in the conceptualization. and measurement of religion and spirituality. American Psychologist, 58, 64-74.

Holland, J.C., Passik, S., Kash, K.M., Russak, S.M., Gronert, M.K., Sison, A., et al.(1999). The role of religious and spiritual beliefs in coping with malignant melanoma. Psycho-Oncology, 8, 14-26.

Holt, C.L., Caplan, L., Schulz, E., Blake, V., Southward, P., Buckner, A. et al. (2009). 
Role of religion in cancer coping among African Americans: A qualitative examination. Journal of Psychosocial Oncology, 27, 248-273.

Hood, R.W., Hill, P.C, \& Williamson, W.P. (2005). The psychology of religious fundamentalism. New York: Guilford Prress.

Hughes, J.W., 'Tomlinson, A., Blumenthal, J.A., Davidson, J., Sketch, M.H., \& Watkins, L.L. (2004). Social support and religiosity as coping strategies for anxiety in hospitalized cardiac patients. Annals of Behavioral Medicine, 28, 179-185.

Hunsberger, B., Owusu, V., \& Duck, R. (1999). Religion and prejudice in Ghana and Canada: Religious fundamentalism, right-wing authoritarianism, and attitudes toward homosexuals and women. International Journal for the Psychology of Religion, 9, 181-94.

Janoff-Bulman, R. (2004). Posttraumatic growth: Three explanatory models. Psychological Inquiry 15, 30-34.

Jenkins, R.A., \& Pargament, K.I. (1988). Cognitive appraisals in cancer patients. Social Science and Medicine, 26, 625-633.

Johnson, R.B. (1997). Examining the validity structure of qualitative research. Education, $118,282-292$.

Kaplar, M.E., Wachholtz, A.B., \& O’Brian, W.H. (2004). The effect of religious and spiritual interventions on the biological, psychological, and spiritual outcomes of oncology patients: A meta-analytic review. Journal of Psychosocial Oncology, $22,39-48$.

Kappeli, S. (2000). Between suffering and redemption: Religious motives in Jewish and Christian cancer patients' coping. Scandinavian Journal of Caring Science, 14, 
82.-88.

Katz, R.C., Flasher, L., Cacciapaglia, H., \& Nelson. S. (2001). The psychosocial impact of cancer and lupus: $A$ cross-validaiional study that extends the generality of "benefit-finding" in patients with chronic disease. Journal of Behavioral Medicine: 24, 561-571.

Kesselring, A., Dodd, M.J., Lindsey, A.M., \& Strauss, A.L. (1986). Attitudes of patients living in Switzerland about cancer and its treatment. Cancer Nursing, 9, 77-85.

King, D.G. (1990). Religion and health relationships: A review. Journal of Religion and Health, 29, 101-112.

Koenig, H.G. (2006). In the wake of a disaster: Religious responses to terrorism and catastrophe. Philadelphia: Templeton Press.

Koenig, H.G. (1997). Is religion good for your heatth? The effects of religion and physical and mental health. New York: Haworth Press.

Koenig, H.G., George, I..K., \& Siegler, I.C. (1988). The use of religion and other emotion-regulating coping strategies amorig older adults. Gerontologist, 28, 303 310.

Koenig, H.G., McCullough, M.E., \& Larson, D.B. (2001). Handbook of religion and health. Oxford: Oxford University Press.

-Krause, N. (2005). Church based social support andmortality. Journals of Gerontology Series B Psychological Sciences \& Social Sciences, 61, 140-146.

Krause, N. (1998). Neighborhood deterioration, religious coping, and changes in health during late life. Gerontologist, 38, 653-664.

Larson, D.B., Swyers, J.P., \& McCullough, M.E. (Eds.) (1997). Scientific research on 
spirituality and health: A consensus report. Rockville, MD: National Institute for Healthcare Research.

Lepore, S.J., Ragan, J.D., \& Jones, S. (2000). Talking facilitates cognitive emotional processes of adaptation to an acute stressor. Journal of Personality and Social Psychology, 78, 499-508.

Lepore, S.J., \& Revenson, T.A. (2006). Resilience and posttraumatic growth: Recovery, resistance, and reconfiguration. In L.G. Calhoun \& R.G. Tedeschi (Eds.), Handbook of posttraumatic growth: Research and practice (pp. 24-36). Mahwah, NJ: Erlbaum.

Levine, E.G., Aviv, C., Yoo, G., Ewing, C., \& Au, A. (2009). The benefits of prayer on mood and well-being of breast cancer survivors. Support Care Cancer, 17, 295 306.

Lincoln, Y.S., \& Guba, E.G. (1985). Naturalistic Inquiry. Newbury Park: Sage Publications.

Loewenthal, K. (1995). Mental Health and Religion. London: Chapman \& Hall. Manne, S., Ostroff, J., Winkel, G., Goldstein, L., Fox, K., \& Grana, G. (2004). Posttraumatic growth following breast cancer: Patient, partner and couple perspectives. Psychosomatic Medicine, 66, 442-454.

Marshall, C., \& Rossman, G.B. (2006). Designing qualitative research $\left(4^{\text {th }} \mathrm{Ed}\right)$. Thousand Oaks, CA: Sage.

Maton, K.I. (1989). The stress-buffering role of spiritual support: Cross-sectional and prospective investigations. Journal for the Scientific Study of Religion, 28, 310323. 
Mattis, J. (2002). Religion and spirituality in the meaning-making and coping experiences of African American women: A qualitative analysis. Psychology of Women Quarterly, 26, 309-321.

Meichenbaum, D. (1985). Siress inoculation training. New York: Pergamon.

Meraviglia, M.G. (2006). Effects of spirituality in breast cancer survivors. Oncology Nursing Forum, 33, E1-E7.

Meraviglia, M.G. (2004). The effects of spirituality on well-being of people with lung cancer. Oncology Nursing Forim, 31, 89-94.

Meraviglia, M.G. (2002). Prayer in people with cancer. Cancer Nursing, 25, 326-331.

Merriam, S.B. (2002). Introduction to qualitative research. In S.B. Merriam (Ed.), Qualitative research in practice: Examples for discussion and analysis. San Francisco, CA: Jossey-Bass.

McConnell, K.M., Pargament, K.I., Ellison, C.G., \& Flannelly, K.J. (2006). Examining the links between spiritual struggles and symptoms of psychopathology in a national sample. Journal of Clinical Psychology, 62, 1469-1484.

McCullough, M.E., Hoyt, W.T., Larson, D.B., Koenig, H.G., \& Thoresen, C. (2000). Religious involvement and mortality: A meta-analytic review. Health Psychology, $165,838-844$.

McCullough, M.E., Larson, D.B., Hoyt, W.T., \& Koenig, H.G. (2000). Religious involvement and mortality: A meta-analytic review. Health Psychology, 19, 211 222.

McGrath, J.E., \& Johnson, B.A. (2003). Methodology makes meaning: How both qualitative paradigms shape evidence and its interpretation. In P.M. Camic, J.E. 
Rhodes, \& L. Yardley (Eds.). Qualitative research in psychology: Expanding perspectives in methodology and design (pp. 31-48). Washington, DC: American Psychological Association.

McGregor; B.A., Antoni; Mi.H., Boyers, A., Ailferi, S.M., Biomberg, B.B., \& Carver, C.S. (2004). Cognitive-behavioral stress management increases benefit finding and immune functioning among women with early-stage breast cancer. Journal of Psychosomatic Research, 56, 1-8.

McLaughlin, B. R. (1994). Devastated spirituality: The impact of clergy sexual abuse on the survivor's relationship with God and the church. Sexual Addiction and Compulsivity, 1, 145-158.

McMillen, C., Zuravin, S., \& Rideout, G. (1995). Perceived benefit from child abuse. Journal of Consulting and Clinical Psychology, 63, 1037-1043.

Miller, W.R., \& Thoresen, C. (2003). Spirituality, religion, and health: An emerging research field. American Psychologist, 58, 24-35.

Mills, B., \& Turnbuil, G. (2004). Broken hearts and mending bodies: The impact of trauma on intimacy. Sexual and Relationship Therapy, 19, 265-289.

Mirola, W.A. (1999). A refuge for some: Gender differences in the relationship between reiigious involvement and depression. Sociology of Religion, 60, 419-437.

Morgan, D.L. (1997). Focus groups as qualitative research ( $2^{\text {nd }}$ ed.). Thousand Oaks, CA: Sage.

Morrow, S.L. (2007). Qualitative research in counseling psychology: Conceptual foundations. The Counseling Psychologist, 35, 209-235. 
Morrow; S.I.., \& Smith, M E. (2000). Qualitative research for counseling psychology. In S.D. Brown, \& R. N Lent (Eds.), Fiandbook of counseling psychology (pp. 199 230). New York: John Willey \& Suns.

Moschella; V.D., Pressman, K.R., Pressman, P., \& Weissman, D.E. (1997). The problem of theodicy and religious response io cancer Journal of Religion and Health, 36, 17-20.

Murray, T.S., Malcarne, V.I., \& Goggin, K. (2003) Alcohol related god/higher power control beliefs, locus of control, and recovery within an alcoholics anonymous paradigm. Alcoholism Treatment Quarterly, 21, 23-39.

National Cancer Institute. (2007). Retrieved February 23, 2007, from http://www.cancer.gov

Neimeyer, R.A. (1993). Constructivism and the cognitive psychotherapies: Some conceptual and strategic contrasts. Journal of Cognitive Psychotherapy, 7, 159171.

Neimeyer, R.A. (2006). Re-storying loss: Fostering growth in the posttraumatic narrative. In L.G. Calhoun \& R.G. Tedeschi (Eds.), Handbook of posttraumatic growth: Research and practice (pp. 68-80). Mahwah, NJ: Erlbaum.

Oren, L.., \& Possick, C. (2009). Religiosity and posttraumatic stress following forced relocation. Journal of Loss \& Trauma, 14, 141160.

Faloutzian, R.F., \& Park, C.L. (2005). Integrative themes in the current science of the psychology of religion. In Paloutzian, R.F., \& Park, C.L. (Eds.), Handbook of the psychology of religion and spirituality (pp. 3-20). New York: Guilford Press. 
Parappully, J., Rosenbaum, R., van den Daele, L., \& Nzewi, E. (2002). Thriving after . . trauma: The experience of parents of murdered children. Journal of Humanistic Psychology, 42,33-70.

Pargament, K.I. (1997). The psychology of religion and coping: Theory, research, practice. New York: Guilford Press.

Pargament, K.I. (2002). The bitter and the sweet: An evaluation of the costs and benefits of religiousness. Psychological Inquiry, 13, 168-181.

Pargament, K.I. (2005). The sacred search for significance: Religion as a unique process. Journal of Social Issues, 61, 665-687.

Pargament, K.I., \& Ano, G.G. (2006). Spiritual resources and struggles in coping with medical illness. Southern Medical Journal, 99, 1161-1162.

Pargament, K.I., Kennell, J., Hathaway, W., Grevengoed, N., Newman, J., \& Jones, W. (1988). Religion and the problem-solving process: Three style of coping. Journal for the Scientific Study of Religion, 27, 90-104.

Pargament, K.I., Ensing, D.S., Falgout, K., Olsen, H., Reilly, B., Van Haitsma, K., \& Warren, R. (1990). God help me: Religious coping efforts as predictors of the outcomes to significant negative life events. American Journal of Community Psychology, 15, 269-286.

Pargament, K.I., Koenig, H.G., \& Perez, L.M. (2000). The many methods of religious coping:Development and initial validation of the RCOPE. Journal of Clinical Psychology, 56, 519-543.

Pargament, K.I., Zinnbauer, B.J., Scott, B.A., Butter, E.M., Zerowin, J., \& Stanik, P. 
(1998). Red flags and religious coping: Identifying some religious warning signs among people in crisis. Journal of Clinical Psychology, 54, 77-89.

Park, C.L. (2004). The notion of growth following stressful life experiences: Problems and prospects. Psychological inquiry, 15, 69-76.Park, C.L. (2005). Reîigion as meaning making framework in coping with life stress. Journal of Social Issues, $61,707-729$.

Park, C.L. (2005). Religion as a meaning-making framework in coping with life stress. Journal of Social Issues, 61, 707-729.

Park, C.L. (1998). Stress-related growth and thriving through coping: The roles of personality and cognitive processes. Journal of Social Issues, 54, 267-277.

Park, C.L., \& Ai, A.L. (2006). Meaning making and growth: New directions for research on survivors of trauma. Journal of Loss and Trauma, 11, 389-407.

Park, C.L., Cohen, L.H., \& Murch, R. (1996). Assessment and prediction of stress related growth. Journal of Personality, 64, 71-105.

Park, C.L., Edmondson, D., Fenster, J.R., \& Blank, T.O. (2008). Meaning making and psychological adjustment following cancer: The mediating roles of growth, life meaning, and restored just-world beliefs. Journal of Consulting and Clinical Psychology, 76, 863-875.

Park, C.L., \& Lechner, S.C. (2006). Measurement issues in assessing growth following stressful life experiences. In L.G. Calhoun \& R.G. Tedeschi (Eds.), Handbook of posttraumatic growth: Research and practice (pp. 3-23). Mahwah, NJ: Erlbaum.

Parry, C., \& Chesler, M.A. (2005). Thematic evidence of psychosocial thriving childhood cancer survivors. Qualitative Health Research, 15, 1055-1075. 
Patton, M.Q. (1990). Qualitaitve evaluation and research methods. Newbury Park, CA: Sage Publications.

Pennebaker, J.W. (1997). Opening up: The healing power of expressing emotions. New York: Guilford Press.

Plante, T.G., \& Boccaccini, M.T. (1997). The Santa Clara strength of religious faith questionnaire. Pastoral Psychology, 45, 375-387.

Poloma, M.M., \& Pendleton, B.F. (1991). The effects of prayer and prayer experiences on measures of general well-being. Journal of Psychology and Theology, 19, 7183.

Richards, P. S., \& Bergin, A. E. (2005). A spiritual strategy for counseling and psychotherapy. Washington, DC: American Psychological Association.

Ringdal, G.I. (1996). Religiosity, quality of life, and survival in cancer patients. Social Indicators Research, 38, 193-211.

Rossetti, S. (1995). The impact of child sexual abuse on attitudes toward God and the Catholic Church. Child Abuse and Neglect, 19, 1469-1481.

Rowatt, W.C., Franklin, L.M., \& Cotton, M. (2005). Patterns and personality correlates of implicit and explicit attitudes towards Christians and Muslims. Journal for the Scientific Study of Religion, 44, 29-43.

Rustoen, T., \& Wilklund, I. (2000). Hope in newly diagnosed patients with cancer. CancerNursing, 23, 214-219.

Sattler, D.N., de Alvarado, A.M., de Castro, N.B., Van Male, R., Zetino, A.M., \& Vega, R. (2006). El Salvador earthquakes: Relationships among acute stress disorder 
symptoms, depression, traunatic event exposure, and resource loss. Journal of Traumatic Siress. 19, 879-893

Schauben, L. J., \& Frazier, P. A. (1995). Vicarious trauma: The effects on female counseiors of working with sexual violence survivors. Psychology of Women Quarterly, 19, 49-64.

Schroevers, M.J., Ranchor, A.V., \& Sanderman, R. (2n04). The role of age at the onset of cancer in relation to survivor' long-term adjustment: A controlled comparison over an eight-year period Psychc-Oncology, 13, 740-752.

Schulz, U., \& Mohamed, N.E. (2004). Turning the tide: Benefit finding after cancer surgery. Social Science and Medicine, 59, 653-662.

Schuster, M.A., Stein, B.D, Jaycox, L.H., Collins, R.L., Marshall, G.N., Elliot, M.N, et al. (2001). A national survey of stress reactions after the September $11^{\text {th }}, 2001$ terrorist attacks. New England Journal of Medicine, 345, 1507-1512.

Sears, S.R., Stanton, A.L., \& Danoff-Burg, S. (2003). The yellow brick road and the emerald city: Benefit-finding, positive reappraisal coping, and posttraumatic growth in women in early-stage breast cancer. Health Psychology, 22, 487-497.

Seligman, M.E.P. (2005). Positive psychology, positive prevention, and positive therapy. In C.R. Snyder \& S.L. L.opez (Eds.), Handbcok of Positive Psychology (pp. 3-9). Oxford: Oxford University Press.

Sethi, S., \& Seligman, M.E.P. (1994). The hope of funcamentalists. Psychological Science, 5,58-61.

Shapiro, D.H., Schwarz, C.E., \& Austin, J.A. (1997). Controlling ourselves, controlling 
our world: Psychology's role in understanding positive and negative consequences of seeking and gaining control. American Psychologist, 51, $1213-$ 1230.

Shaw, A., Joseph, S., \& Linley, P.A. (2005). Religion, spirituality, and posttraumatic growth: A systematic review. Mental Health, Religion, and Culture, 8, 1-11.

Sidran Institute for Traumatic Stress Education and Advocacy. (2006). Retrieved April 11, 2006, from http://www.sidran.org/whatistrauma.html.

Siegel, K., \& Schrimshaw, E.W. (2000). Perceiving benefits in adversity: Stress related growth in women living with HIV/AIDS. Social Science and Medicine, 51, 15431544.

Silberman, I. (2005). Religion as a meaning system: Implications for the new millennium. Journal of Social Issues, 61, 641-664.

Smith, S.G. \& Cook, S.L. (2004). Are reports of posttraumatic growth positively biased? Journal of Traumatic Stress, 17, 353-358.

Sofaer, S. (1999). Qualitative methods: What are they and why use them? Health Services Research, 34, 1101-1118.

Somali, A.M., Heckman, T.G. (2000). Correlates of spirituality and well-being in a community sample of people living with HIV disease. Mental Health, Religion, \& Culture, 3, 57-70.

Spilka, B., Hood, R.W., Hunsberger, B., \& Gorsuch, R.L. (2003). The psychology of religion: An empirical approach ( $3^{\text {rd }}$ ed.), New York: The Guilford Press.

Stanton, A.L., Bower, J.E, \& Low, C.A. (2006). Posttraumatic growth after cancer. In 
L.G.Calhoun \& R.G. Tedeschi (Eds.), Handbook of posttraumatic growth: Research and praciice (pp. 138-175). Mahwah, NJ: Erlbaum.

Stark, R. (1.996). Religion as context: Hellfire and delinquency one more time. Sociology of Religion, $57,163-173$.

Steinhauser, K.E., Voils, C.I., Clipp, E.C., Bosworth, H.B., Christakis, N.A., \& Tulsky, J.A. (2006). Are you at peace: One item to probe spiritual concerns at the end of life. Archives of Internal Medicine, 166, 101-105.

Stone, H.W., Cross, D.R., Purvis, K.B., \& Young, M.L. (2003). A study of the benefit of social and religious support on church members during times of crisis. Pastoral Psychology, 51, 327-340.

Tarakeshwar, N., Vanderwerker, L.C., Paulk, E., Pearce, M.J., Kasl, S.V., \& Prigerson, H.G. (2006). Religious coping is associated with the quality of life of cancer patients with advanced cancer. Journal of Palliative Medicine, 9, 646-657.

Tatsumura, Y., Maskarinec, G., Shumay, D.M., \& Kakai, H. (2003). Religious and spiritual resources, CAM, and conventional treatment in the lives of cancer patients. Alternative Therapies, 9, 64-71

Taylor, E.J., \& Outlaw, F.H. (2002). Use of prayer among persons with cancer. Holistic Nursing Practice, 16, 46-60.

Tedeschi, R.G., \& Calhoun, L.G. (1995). Trauma and transformation: Growing in the aftermath of suffering. Thousand Oaks, CA: Sage.

Tedeschi, R.G., \& Calhoun, L.G. (1996). The posttraumatic growth inventory: Measuring the positive legacy of trauma. Journal of Traumatic Stress, 9, 455-471.

Tedeschi, R.G., \& Calhoun, L.G. (2004). Posttraumatic growth: Conceptual foundations 
and empirical evidence. Psychological Inquiry, i.5, 1-18.

Thornton, A.A., Perez, M.A., \& Meyerowitz, B.E. (2005). Posttraumatic growth in prostate cancer patients and their partners. Unpublished data.

Tomich, P.L., \& Helgelson, V.S. (2002). Five years later: A cross-sectional comparison of breast cancer survivors and healthy women. Psycho-Oncology, 11, 154-169.

Tomich, P.L., \& Helgeson, V.S. (2004). Is finding something good in the bad always good? Benefit finding among women with breast cancer. Health Psychology, 23, $16-23$.

Tsang, J., \& McCullough, M.E. (2003). Measuring religious constructs: A hierarchical approach to construct organization and scale selection. In S.J. Lopez \& C.R. Snyder (Eds.) Positive psychological assessment: A handbook of models and measures (pp. 345-360). Washington, D.C.: American Psychological Association.

Urcuyo, K.R., Boyers, A.E., Carver, C.S., \& Antoni, M.H. (2005). Finding benefit in breast cancer: Relations with personality, coping, and concurrent well-being. Psychology and Health, 20, 175-192.

Valentine, L., \& Feinauer, L. L. (1993). Resilience factors associated with female survivors of childhood sexual abuse. American Journal of Family Therapy, 21, $216-224$.

Vis, J.A., \& Boynton, H.M. (2008). Spirituality and transcendent meaning making: Possibilities for enhancing posttraumatic growth. Journal of Religion and Spirituality in Social Work, 27, 69-86.

Wallston, K. A., Malcarne, V. L., Flores, L., Hansdottir, I., Smith, C.A., Stein, M.J., Weisman, M.H., \& Clements, P.J. (1999). Does God determine your health? The God 
Locus of Heath Conirol scale. Cogritive Therapy and Research, 23, $13 \mathrm{i}-142$.

Weiss, T. (2004). Correlates of postraumatia growth in husbands of breast cancer survivors. Psycho-oncology, 13, 260-268.

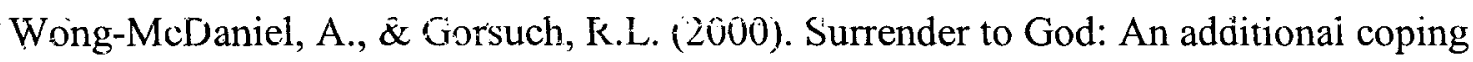 style. Journal of Psychology and Theology, 28, 149-161.

Worthen, V.E., \& McNeill, 3.W. (200\%). A phenomenological investigation of "good" supervision events in S.B. Merriam (Ed.), Qualitative research in practice: Examples for discussion and analysis. San Francisco, CA: Jossey-Bass.

Wortman, C.B. (2004). Posttraumatic growth: Progress and problems. Psychological Inquiry, 15,81-90.

Yalom, E. (1980). Existential Psychotherapy. New York: Basic Books.

Yarhouse, M.A. (2003). Ethical issues in considering 'religious impairment' in diagnosis. Mental Health, Religion, and Cuiture, 6, 131-147.

Zinnbauer, B.J., \& Pargament K.I. (2005). Religiousness and spirituality. In R.F. Paloutzian \& C.L. Park (Eds.), Handbook of the psychology of religion and spirituaiity (pp. 21-42). New York: Guilford Press. 